\title{
Random decrement technique for detection and characterization of nonlinear behavior
}

\author{
Krautwald Vesterholm, Karsten; Brincker, Rune; Brandt, Anders
}

Published in:

Mechanical Systems and Signal Processing

Link to article, DOI:

10.1016/j.ymssp.2020.106841

Publication date:

2020

Document Version

Early version, also known as pre-print

Link back to DTU Orbit

Citation (APA):

Krautwald Vesterholm, K., Brincker, R., \& Brandt, A. (2020). Random decrement technique for detection and characterization of nonlinear behavior. Mechanical Systems and Signal Processing, 143, [106841].

https://doi.org/10.1016/j.ymssp.2020.106841

\section{General rights}

Copyright and moral rights for the publications made accessible in the public portal are retained by the authors and/or other copyright owners and it is a condition of accessing publications that users recognise and abide by the legal requirements associated with these rights.

- Users may download and print one copy of any publication from the public portal for the purpose of private study or research.

- You may not further distribute the material or use it for any profit-making activity or commercial gain

- You may freely distribute the URL identifying the publication in the public portal

If you believe that this document breaches copyright please contact us providing details, and we will remove access to the work immediately and investigate your claim. 


\title{
Random decrement technique for detection and characterization of nonlinear behavior
}

\author{
Karsten Krautwald Vesterholm ${ }^{\mathrm{a}, *}$, Rune Brincker ${ }^{\mathrm{b}}$, Anders Brandt ${ }^{\mathrm{a}}$ \\ ${ }^{a}$ University of Southern Denmark, Department of Technology and Innovation, Campusvej 55, DK-5230 Odense M, Denmark \\ ${ }^{b}$ Technical University of Denmark, Department of Civil Engineering, Anker Engelunds Vej 1, DK-2800 Kgs. Lyngby, \\ Denmark
}

\begin{abstract}
When performing operational modal analysis, the mechanical system being investigated is assumed to be linear. If this assumption is not true, errors could be made when estimating the modal parameters of the system. There are no generally accepted robust analysis procedures available that can verify or validate the assumption of linearity in an output-only setting. In the present study, a method of detecting nonlinear behavior from a random response signal is proposed. The method is based on the random decrement (RD) technique, which involves identifying triggering points (TPs) in a measured response signal. The proposed procedure is to identify a set of TPs, where each member of the set represents a unique initial condition. A $\mathrm{RD}$ signature is then calculated for each set member of TPs. For the response of a linear system, all RD signatures are equal regardless of the initial conditions. For a nonlinear system, not all RD signatures will be equal for different initial conditions. Detection of nonlinear behavior is then possible by using principal component analysis and a parameterization of RD signatures to analyze the sameness of all the RD signatures in the set. The proposed analysis procedure is investigated with numerical and experimental test cases of a single-degree-of-freedom stick-slip system. Results show that it is possible to distinguish between linear and nonlinear behavior using this method. It is also possible to characterize which aspect of the system the nonlinear behavior relates to.
\end{abstract}

Keywords: detection of nonlinearity, random decrement, random vibration, output-only, single-degree-offreedom

\section{Introduction}

The investigation of modal parameters of structures under ambient vibration is known as operational modal analysis (OMA). In OMA, only the response of a structure is measured. The input force that caused the vibration is not measured. There can be many reasons why the input force is not measured. Often it is simply because it is impossible to perform such measurement. This could be the case of a road bridge being excited by traffic, an offshore structure excited by wind and ocean waves, or a plane in flight excited by aerodynamic forces. This type of vibration analysis can be done for many reasons. It could be to verify an analytical model, to perform model updating, to monitor the condition of a structure, or to identify its modal parameters.

One of the fundamental assumptions of OMA is that the system under investigation is linear [1. This is however not always the case. In fact most structures will exhibit nonlinear behavior to some extent when under certain levels of excitation. This means that the present OMA techniques might give erroneous results when estimating the modal parameters of a structure exhibiting nonlinear behavior. Many types of nonlinearities exist, such as; friction, hysteretic material properties, geometric nonlinearity, contact nonlinearity

\footnotetext{
${ }^{*}$ Corresponding author

Email addresses: kav@iti.sdu.dk (Karsten Krautwald Vesterholm), runeb@byg.dtu.dk (Rune Brincker), abra@iti.sdu.dk (Anders Brandt)
} 
from boundary conditions, to name a few. There are several research fields and industries where structures of interest have nonlinearities, in civil engineering, mechanical engineering, aerospace, automotive, ect.

In experimental modal analysis (EMA), where both the input force(s) and the response(s) are measured, a test for linearity is a standard procedure when a shaker is used to excite the structure. The procedure is to use different input force levels to excite the structure. If the frequency response functions (FRFs) are equal for the different force levels, the structure is considered linear 2. If the FRFs are not equal, that is an indication of a nonlinearity in the system. A similar test is not possible in OMA, since the input force is not measured, and the FRF cannot be computed.

An OMA analysis can be performed in the time domain or the frequency domain. When using the time domain approach, a correlation (covariance) function matrix is estimated from the measured responses. From the correlation function matrix, modal parameters are estimated using well known time domain modal parameter estimation methods such as covariance driven stochastic subspace identification (COVSSI), Ibrahim Time Domain (ITD), Eigensystem Realization Algorithm (ERA), Least Squares Complex Exponential (LSCE), Polyreference Time Domain (PTD), etc. Further details about how the OMA analysis is performed are described by Brincker and Ventura [1].

Estimating the correlation function matrix can be done in more than one way. A commonly used estimate of the correlation function between signals $x(t)$ and $y(t)$ is given by Bendat and Piersol [3]

$$
\hat{R}_{y, x}(\tau)=\frac{1}{T} \int_{0}^{T} x(t) y(t+\tau) d t 0 \leq \tau<T
$$

When using this estimator for the correlation function matrix, it is here referred to as the standard OMA analysis.

A study have been conducted by Friis et al. 4 where the effect of applying the standard OMA analysis on a system known to be nonlinear is investigated. The study concludes that for their numerical system, OMA techniques are able to extract modal information about the underlying linear system. However, the estimates are concluded to be biased and have a higher variability of both frequency and damping estimates in the presence of a nonlinearity. As shown by their study, current OMA techniques are known to give less accurate results for nonlinear systems. Their result is a motivator for the present study, as this is a step towards improving OMA techniques when applied to nonlinear systems.

In the field of nonlinear system identification, there are well known techniques for detecting the presence of nonlinearities, as reviewed by Kerschen et al. [5]. Common for all these techniques is the fact that both input force and the response must be measured. When EMA or nonlinear system identification are inapplicable, an alternative method of detection if the structure is linear or nonlinear is needed. The present study proposes such a method. Inspiration to the proposed method is taken from the field of nonlinear system identification. Detecting whether a nonlinearity is present or not, is the first step in the nonlinear system identification process, as stated by Kerschen et al. [5]. This step is replicated in an OMA setting, and a procedure to perform it is proposed in the present study. Knowing if a system exhibits nonlinear behavior is of great value when performing OMA. If the system is found to exhibit nonlinear behavior, the standard OMA analysis should either be applied with caution or methods capable of dealing with the nonlinearity should be applied instead.

Instead of the correlation function matrix, random decrement (RD) signatures can be used to estimate the modal parameters. RD signatures are calculated using the RD technique and can be treated as correlation functions. The RD technique is the foundation of the analysis procedure that is proposed in the present study.

RD was first presented by Cole in the late 1960s and early 1970s, 6, 7, 8, as a method of obtaining correlation function estimates of a signal while the signal was being measured. Vandiver et al. [9] gave a thorough mathematical background for the technique applied on single-degree-of-freedom systems (SDOF) excited by zero mean Gaussian white noise. In the beginning only auto RD signatures were calculated, meaning only the diagonal of the RD signature matrix can be computed. When Ibrahim [10] proposed how to compute cross RD signatures, it allowed for the cross terms to calculated as well. With the arrival of the cross $\mathrm{RD}$ signature, the foundation of the $\mathrm{RD}$ technique was laid for Asmussen to write his $\mathrm{PhD}$ thesis 
11] fully developing the RD technique to be used in modal analysis of linear structures. Presently, the RD technique is commonly applied in the field of OMA [1].

Using OMA with the RD technique on nonlinear structures has been investigated in several studies, 12, 13, 14, 15, 16, 17, 18. The nonlinearity is typically described in the form of a response amplitude dependency of modal parameters. Common for most studies of OMA with the RD technique applied to nonlinear structures is that the presence of a nonlinearity is assumed, and the objective is to characterize if the nonlinearity is damping or frequency related. These previous studies have a scientific weakness in the fact that their purpose is to confirm an assumption of nonlinearity and neglecting an investigation of other possible causes of the observations. A stronger scientific approach would be to first question the assumption, whether the structure exhibits nonlinear behavior. This question is considered the null hypothesis in the present study. If indications of nonlinear behavior are not present, the null hypothesis must be rejected, and the alternative hypothesis is accepted, i.e. behavior of the structure is interpreted as linear.

Some research has already been carried out to investigate OMA techniques to detect nonlinearities e.g. Marcias et al. [19] and Vesterholm et al. [20]. Both studies take a time domain approach. Marcias et al. studied a method of comparing experimental measurements with a fully developed finite element model (FEM) of a bowstring arch bridge. The method consisted of calculating a subspace representing the time history measurement of the simulated response using FEM and experimental measurement, respectively. The two subspaces were then compared, and deviations between the two were attributed to nonlinear behavior in the experimental structure. This method therefore requires a FEM with which to simulate time history response data. It can also be argued that other causes exist that explain the deviation between the two subspaces. This could be unaccounted differences between the model and actual bridge, or differences in the excitation forces between the model and the actual bridge. The study by Vesterholm et al. 20, is the foundation which the present study builds upon. There, a single-degree-of-freedom system was modeled and a forced response time history simulated. Two systems were compared using the RD technique, a linear, and a nonlinear. A crude distinction in the results of the analysis of the two systems was made that revealed one system to be linear and the other to be nonlinear. The analysis did not require anything a priori known about the system under investigation, other than that it had a random response.

The outcome of the present study is the introduction of a new analysis step in addition to the well known analysis procedure of time domain OMA. The addition consist of a test of linearity, corresponding to the standard EMA test, to be performed before estimating the correlation function matrix.

There are many possible sources of nonlinearities, depending on the type of structure in question. This study tests the SDOF stick slip system, both numerically and experimentally, to demonstrate the proposed method. A system with this nonlinearity is chosen because it exhibits amplitude dependent behavior in both damping and frequency. This is desired in the test case to demonstrate the capabilities of the methods to detected and characterize amplitude dependent behavior in damping, frequency, or both together.

The present study is limited to SDOF systems, developing theory and methodologies for a simple case. Expansion of the methodology to MDOF systems are part of the authors future research.

The analysis procedure presented in the present study is part of the analysis framework known as nonlinearity optimized random decrement (NORD). The framework was first presented by Vesterholm et al. 20, by presenting a simple method of detecting nonlinear behavior. The framework was then expanded upon by Friis et al. [21] by implementing a method of accurately identifying the modal parameters of a friction coupled system. NORD can be considered a strategy of applying the RD technique on nonlinear systems, in order to extract modal parameters in a robust manner in an output-only setting.

\section{Theory}

The mathematical definition of a linear system is where the principle of superposition applies, and is explained as follows. A system with initial conditions $T_{1}=\left\{y_{1}(0), \dot{y}_{1}(0)\right\}$ excited by $x_{1}$ produces response $y_{1}$. In another instance the system is in initial condition $T_{2}=\left\{y_{2}(0), \dot{y}_{2}(0)\right\}$, and the excitation $x_{2}$ produces response $y_{2}$. The system is linear if, and only if, for initial condition $T_{3}=\left\{A y_{1}(0)+B y_{2}(0), A \dot{y}_{1}(0)+B \dot{y}_{2}(0)\right\}$ the excitation $A x_{1}+B x_{2}$ produces the response $A y_{1}+B y_{2}[22$. When a system is assumed linear, this 
principle must always apply. In order to confirm if this is in fact the case, an infinite combination of $A, B$ and $x_{1}, x_{2}$ pairs must be investigated. This is of course impossible in practice. It is somewhat easier to use the principle of superposition to reveal a system to be nonlinear, because only one case where the principle does not hold must be presented.

Presenting the aspects of a mechanical system that are investigated in the present study, a starting point is taken with the homogeneous ordinary differential equation (ODE) governing the motion of a linear SDOF system, which is given as

$$
m \ddot{y}(t)+c \dot{y}(t)+k y(t)=0
$$

For an underdamped system $\left(c^{2}<4 m k\right)$, the general solution to the ODE is

$$
y(t)=C e^{-\alpha t} \cos (\omega t-\phi), \alpha=\frac{c}{2 m}, \omega=\sqrt{\frac{k}{m}-\frac{c^{2}}{4 m^{2}}}
$$

where $C$ is some constant. This solution is an exponentially decaying cosine, also called the free decay of the system. $\alpha$ and $\omega$ are defined from $m, c, k$, mass, damping and stiffness entirely. This means that for all initial conditions, damping and frequency will be the same for a linear SDOF system.

The equation governing the motion of an SDOF system with nonlinear damping and stiffness is given as

$$
m \ddot{y}(t)+f_{n l}(\dot{y}(t), y(t))=0
$$

This equation does not have a general solution. Only for special cases does the nonlinear ODE have a general solution, and these are of no practical use in the present study. Since every type of nonlinearity can be considered a special case of Equation (4), it is impossible to generally describe what influence the nonlinearities have on the frequency and damping of the free decay. It is considered reasonable to assume that the free decay of a nonlinear SDOF system will appear similar to an exponentially decaying sine wave. However, since the stiffness and damping are now functions of the displacement and velocity of the system, frequency and damping have become dependent on the initial conditions [23. Changes in frequency and damping of the free decay are aspects of the SDOF system that will be analyzed to detect if system behavior is linear or nonlinear.

The proposed detection procedure is to use the RD technique to investigate how a system behaves using a set of initial conditions. Based on an analysis of the frequency and damping of the system at the set of initial conditions, it may be determined whether the principal of superposition applies or not.

\subsection{Linear SDOF system under random excitation}

The detection procedure proposed in the present study applies only when the mechanical SDOF system is excited by an unknown random signal. For the case where a linear SDOF system is excited by a stationary, white, Gaussian, zero mean force, the autocorrelation is exactly proportional to the free decay from a specific initial displacement, and zero initial velocity 9. This is expressed as the non homogeneous equation of motion

$$
m \ddot{y}(t)+c \dot{y}(t)+k y(t)=F(t)
$$

where $F(t)$ is the force applied to the system. The autocorrelation function of the response is then calculated as 3 .

$$
R_{y, y}(\tau)=E[y(t) y(t+\tau)]
$$

For a nonlinear system, the autocorrelation of the response is also calculated as in Equation (6), and if the input force is known, information about the nonlinearity can determined as several cases shows in [24]. However, without the information of the input force, nonlinear behavior cannot be determined from the autocorrelation. 


\subsection{Random decrement technique}

The RD technique will in this section be described in some detail to understand its most common use, and give a foundation upon which to build the method developed in the present study.

Vandiver et at. 9] described the RD signature as an average of a collection of segments gathered from a common time-history. All segments in this averaging have a common specific initial condition. In RD theory the initial condition of the segments are determined by the triggering condition (TC). The time samples in the discrete response signal that satisfy the TC are known as the triggering points (TPs). For a mechanical system the amplitude and slope of the response signal at the TP is interpreted physically as the initial displacement and velocity of the system respectively. Applying a specific TC in RD theory is therefore equivalent to applying a specific initial condition. Vandiver et al. [9] was the first to express the $\mathrm{RD}$ signature as a conditional mean, which relates it to the correlation function

$$
D_{y, y}(\tau)=E\left[y(t+\tau) \mid T_{y(t)}\right]
$$

The estimate of this expression is the original RD estimator introduced by Cole $[6$

$$
\hat{D}_{y, y}(\tau)=\frac{1}{N} \sum_{l=1}^{N} y\left(t_{l}+\tau\right) \mid T_{y\left(t_{l}\right)}
$$

where $y(t)$ is the discrete vibration signal being analyzed and $T_{y\left(t_{l}\right)}$ describes the triggering condition. $N$ time samples $t_{l}$ are identified in the signal $y(t)$ that satisfies the TC, and are therefore identified as TPs.

The term in the RD calculation that controls the initial condition properties of the resulting RD signature is the TC $T_{y\left(t_{l}\right)}$. The general triggering condition was introduced by Brincker et al. [25, and is given as

$$
T_{y(t)}^{G T}=\left\{a_{1} \leq y(t)<a_{2}, b_{1} \leq \dot{y}(t)<b_{2}\right\}
$$

where $a_{1}$ and $a_{2}$ can be any value between the minimum and maximum of the signal values and form a band in the signal. Similarly, $b_{1}$ and $b_{2}$ can take any values between the minimum and maximum of the derivative of the signal, also forming a band in the derivative of the signal. For a linear system excited by a zero mean, stationary, Gaussian force, the relationship between the RD signature using the general triggering condition and the autocorrelation function of the response was derived by Asmussen [11], and is given by

$$
\begin{gathered}
D_{y, y}(\tau)=\frac{R_{y, y}(\tau)}{\sigma_{y}^{2}} \cdot \tilde{a}-\frac{\dot{R}_{y, y}(\tau)}{\sigma_{\dot{y}}^{2}} \cdot \tilde{b} \\
\tilde{a}=\frac{\int_{a_{1}}^{a_{2}} y p_{y}(y) d y}{\int_{a_{1}}^{a_{2}} p_{y}(y) d y}, \tilde{b}=\frac{\int_{b_{1}}^{b_{2}} \dot{y} p_{\dot{y}}(\dot{y}) d \dot{y}}{\int_{b_{1}}^{b_{2}} p_{\dot{y}}(\dot{y}) d \dot{y}}
\end{gathered}
$$

$\tilde{a}$ is the mean of the signal value for all the TPs in the signal band between $a_{1}$ and $a_{2}$. Similarly, $\tilde{b}$ is the mean of the derived signal at all the TPs in the band between $b_{1}$ and $b_{2} . \tilde{a}$ and $\tilde{b}$ can be considered scaling constants for how much of $D_{y, y}$ is proportional to the symmetric $R_{y, y}$ and the antisymmetric $\dot{R}_{y, y}$. The antisymmetric correlation function is the derivative of the correlation function and is calculated as [3]

$$
\dot{R}_{y, y}(\tau)=E[y(t) \dot{y}(t+\tau)]
$$

From the general TC, specific TCs can be formulated by specifying $a_{1}, a_{2}, b_{1}$, and $b_{2}$ in Equation (9).

\subsubsection{Implementation of the positive point triggering condition}

The standard method of applying the RD technique in OMA, as described by Brincker and Ventura 1, is first to assume linearity in the system under investigation. A specific TC is then chosen to perform the $\mathrm{RD}$ calculation, for instance positive point (PP), which Asmussen [11] defines as

$$
T_{y(t)}^{P P}=\left\{a_{1} \leq y(t)<a_{2},-\infty<\dot{y}(t)<\infty\right\}
$$


Here, TPs are identified when the time series has a value between $a_{1}$ and $a_{2}$. PP can be though of as a generalization of level crossing (LC), which is the original TC proposed by Cole [6] who originally presented the RD technique. There a TP was identified every time the signal crosses some amplitude level $a$. This is different from PP, because when implementing LC a sample has to be chosen for that particular level crossing. If the signal does not have an very high sample rate, this could mean a sample with significantly different amplitude is chosen to represent the level $a$. This does not happen for PP, where only samples belonging to the interval $\left[a_{1}, a_{2}\right]$ are identified as TPs.

After PP has been applied to identify TPs, the next step is to perform the averaging of the segments represented by the TPs, as described in Equation (8). For a linear system excited by a zero mean, stationary, Gaussian force, the relationship between the RD signature using PP and the autocorrelation function of the response is as follows

$$
D_{y, y}(\tau)=\frac{R_{y, y}(\tau)}{\sigma_{y}^{2}} \cdot \tilde{a}, \tilde{a}=\frac{\int_{a_{1}}^{a_{2}} y p_{Y}(y) d y}{\int_{a_{1}}^{a_{2}} p_{Y}(y) d y}
$$

where $\tilde{a}$ is the triggering level, $\sigma_{y}$ is the standard deviation of the response signal $y(t)$, and $p_{Y}$ is the probability distribution of the time series.

PP identifies TPs when the signal has a value in the interval $\left[a_{1}, a_{2}\right]$. The slope of the signal can have any value at the TP. The physical interpretation of this is that a range of initial displacements is specified, but the velocity can take any value. When investigating a linear system, this method is very robust in part because the number of TPs is easily varied, and potentially very high when using this method.

However, when investigating whether systems are exhibiting nonlinear behavior, a more refined strategy for identifying TPs is needed. Here PP is suitable as it allows very specific samples to be identified as TPs.

\subsubsection{Triggering condition for detection}

As mentioned in Section 2, a method for determining a system to exhibit nonlinear behavior, is to verify that the principle of superposition does not apply. The detection procedure developed in the present study is founded in investigating how the system behaves under several different initial conditions. The resulting frequencies and rates of decay are analyzed. For a linear system, different initial conditions will not result in a different frequency or rate of decay. The opposite is true for a system exhibiting nonlinear behavior. The TC in RD theory is equivalent to initial condition.

To realize an analysis procedure capable of investigating many different initial conditions, a TC must be formulated. The TC is developed with the starting point of PP, introduced above, and can be though of a new implementation of the general TC in Equation (9). This is implemented by formulating a set of TCs, which corresponds to a set of initial conditions, in the form

$$
T_{y(t)}^{i, j}=\left\{a_{i} \leq y(t)<a_{i+1}, b_{j}<|\dot{y}(t)|<b_{j+1}\right\}
$$

where $a_{i}$ is a set of $m$ amplitude levels in the signal, and $b_{j}$ is a set of $n$ amplitudes in the absolute value of the derived signal. A way of interpreting this set of $(m-1) \times(n-1)$ TCs is to consider it as two separate steps. First apply PP on $m-1$ amplitude ranges defined by $a_{1}, a_{2}, \cdots a_{m}$. Then, assign the identified TPs at each of the $m-1$ amplitude ranges, to $n-1$ groups depending on which slope range in the interval $\left[b_{j}, b_{j+1}\right]$ the individual TP belongs to. Applying this set of $(m-1) \times(n-1)$ TCs to one signal, is equivalent to investigating the free decay of the system using $(m-1) \times(n-1)$ unique initial conditions.

It is here shown that the resulting RD signature for a linear system will result in exactly the same RD signature as when applying PP. The RD signature found using the new TC can be expressed as a combination of the correlation function, and the differentiated correlation function in the following way

$$
D_{y, y}^{i, j}(\tau)=\frac{R_{y, y}(\tau)}{\sigma_{y}^{2}} \cdot \tilde{a}_{i}-\frac{\dot{R}_{y, y}(\tau)}{\sigma_{\dot{y}}^{2}} \cdot \tilde{b}_{j}+\frac{\dot{R}_{y, y}(\tau)}{\sigma_{\dot{y}}^{2}} \cdot \tilde{b}_{j}
$$

where

$$
\tilde{a}_{i}=\frac{\int_{a_{i}}^{a_{i+1}} y p_{Y}(y) d y}{\int_{a_{i}}^{a_{i+1}} p_{Y}(y) d y}, \tilde{b}_{j}=\frac{\int_{b_{j}}^{b_{j+1}} \dot{y} p_{\dot{y}}(\dot{y}) d \dot{y}}{\int_{b_{j}}^{b_{j+1}} p_{\dot{y}}(\dot{y}) d \dot{y}}
$$




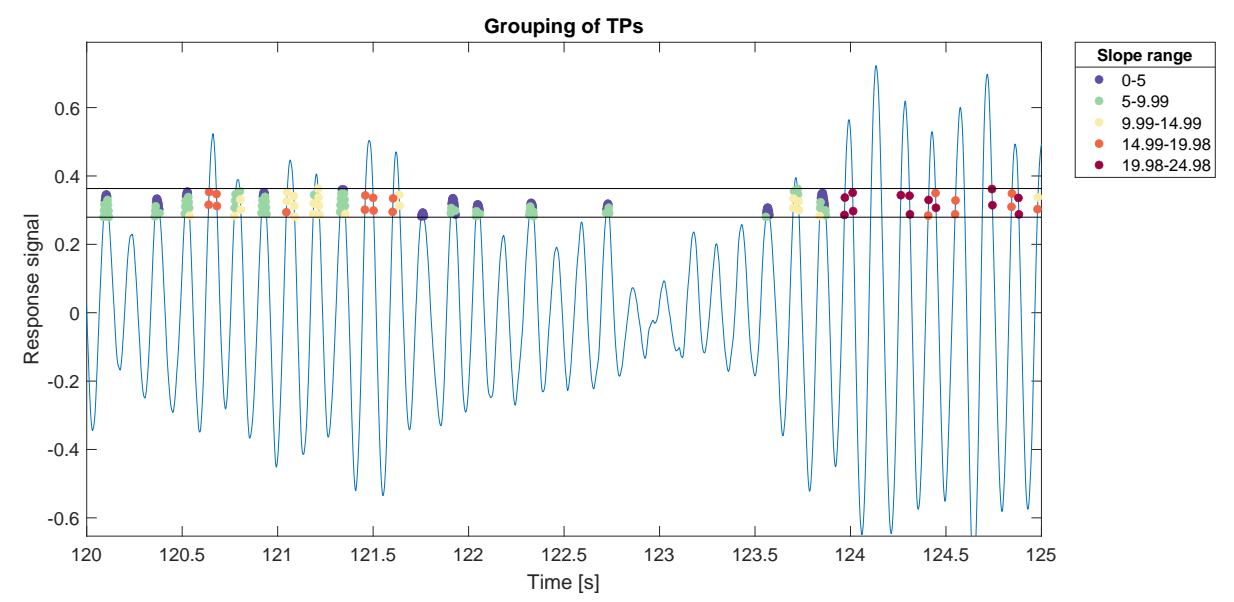

Figure 1: Illustrating use of the triggering condition $T_{y(t)}^{i, j}$ for detection of nonlinear behavior, where triggering points are identified in a triggering bound at $[0.28,0.36]$, illustrated by the horizontal black solid lines, and then assigned to 5 groups defined from the derived signal.

The second and third terms of Equation (16) cancel each other out, and the expression is equal to Equation (14). This means that the application of this new $\mathrm{TC}$ on a linear system will yield the same RD signature as applying the PP TC, regardless of the value of $b_{1}$ and $b_{2}$.

A formulation of how $a_{i}$ and $b_{j}$ are defined based on $y(t)$ and $\dot{y}(t)$ is presented here. $a_{i}$ is defined based on the standard deviation of $y(t)$ such that $a_{i}$ has values in the interval between $0.75 \sigma_{y}$ and $2.2 \sigma_{y}$. This interval is chosen to ensure a high number of TPs are identified at each amplitude. If larger values for $a_{i}$ are chosen, too few TPs will be identified to perform a consistently successful analysis. $b_{j}$ is defined based on the standard deviation of $\dot{y}(t)$ such that $b_{j}$ has values in the interval between 0 and $2 \sigma_{\dot{y}}$. This interval is limited at $2 \sigma_{\dot{y}}$ to ensure that all slope ranges will contain a useful amount of TPs for all $a_{i}$. If $b_{j}$ is too high, there will be no TPs in the desired range for high values of $a_{i}$, because the slope is generally low for high signal magnitudes.

An example of the use of the new TC is presented in Figure 1, with triggering bounds at $\left[a_{1}, a_{2}\right]=$ $[0.28,0.36]$ in the response signal. When all TPs belonging to this triggering level have been identified, they are assigned to 5 groups formed between the 6 linearly spaced amplitudes $\left[b_{1}, \cdots b_{6}\right]=[0,5,10,15,20,25]$ that cover a range of the derived signal. Each group is indicated by a color.

\subsection{Principal Component Analysis of RD signatures}

A key aspect of the detection procedure introduced in the present study is to analyze a set of RD signatures using the principal component analysis (PCA). Based on the set of RD signatures obtained from the response signal using a set of triggering conditions, nonlinear behavior can be detected. The detection of nonlinear behavior is based on the RD signatures in the set being similar or different from each other. The system behavior is interpreted as nonlinear when not all RD signatures are the same within some margin of error, and linear when all RD signatures are the same. As a method of quantifying how different the RD signatures are from each other, PCA is utilized. PCA was introduced by Pearson [26] and Hotelling [27. The outcome of PCA in this setting is quantification of variance in the set of RD signatures, meaning, how much of the variance can be explained by the principal components (PCs). If more than one $\mathrm{PC}$ has to be included to account for the total variance, not all RD signatures contain the same information, and the null hypothesis of nonlinear behavior can be accepted. This means the system is interpreted as exhibiting nonlinear behavior. If $100 \%$ of the total variance in the set of RD signatures can be explained by the first $\mathrm{PC}$, all RD signatures in the set contains the same information. If this is the case, any RD signature in the set is a linear combination the others. For this to be true, $\mathrm{RD}$ signatures in the set can only deviate from each other by a scaling factor, not a difference in frequency or decay rate. Here, the null hypothesis is rejected, and 
the system behavior is interpret as linear. Before performing the PCA, all RD signatures are normalized to z-score, i.e. to have zero mean and unit standard deviation. This normalization is done to standardize units for the PCA. Next step is forming a matrix A from the $(m-1) \times(n-1)$ z-score normalized RD signatures as columns. The order of the columns has no influence on the PCA. Then calculate the covariance matrix $\boldsymbol{\Sigma}$ of $\mathbf{A}$, as

$$
\boldsymbol{\Sigma}=\left[\begin{array}{cccc}
\operatorname{cov}\left(D^{1,1}, D^{1,1}\right) & \operatorname{cov}\left(D^{1,1}, D^{1,2}\right) & \cdots & \operatorname{cov}\left(D^{1,1}, D^{m-1, n-1}\right) \\
\operatorname{cov}\left(D^{1,2}, D^{1,1}\right) & \operatorname{cov}\left(D^{1,2}, D^{1,2}\right) & \cdots & \operatorname{cov}\left(D^{1,2}, D^{m-1, n-1}\right) \\
\vdots & \vdots & \ddots & \vdots \\
\operatorname{cov}\left(D^{m-1, n-1}, D^{1,1}\right) & \operatorname{cov}\left(D^{m-1, n-1}, D^{1,2}\right) & \cdots & \operatorname{cov}\left(D^{m-1, n-1}, D^{m-1, n-1}\right)
\end{array}\right]
$$

where the covariance between two RD signatures with zero mean is defined as

$$
\operatorname{cov}\left(D^{1,1}, D^{1,2}\right)=\frac{1}{N-1} \sum_{k=1}^{N} D^{1,1}(k) D^{1,2}(k)
$$

Notice that the dependency on $\tau$ has been omitted to simplify notation. Next step is to perform and eigenvalue decomposition (EVD) of $\boldsymbol{\Sigma}$.

$$
\boldsymbol{\Sigma}=\sum_{s=1}^{p} \lambda_{s} e_{s} e_{s}^{T}
$$

where $\lambda_{s}$ and $e_{s}$ are the eigenvalues and eigenvectors respectively. $\lambda_{s}$ and $e_{s}$ are then sorted in a ascending order of $\lambda_{s}$, which are all real valued and nonnegative because $\boldsymbol{\Sigma}$ is positive semidefinite. The parameters of interest here are the $\lambda_{s}$, as they represent the variances of the PCs and therefore carry the information of the data. To determine if all RD signatures are the same, the variance explained by each $\mathrm{PC}$ must be calculated. The expression for the variance explained by $\mathrm{PC}_{s}$ is

$$
\eta_{s}=\frac{\lambda_{s}}{\sum_{i=s}^{p} \lambda_{s}}
$$

If all RD signatures in $\mathbf{A}$ are the same, $\eta_{1}=1$, and all others would be 0 . If this is not the case, $\eta_{1}<1$, i.e. not all information in the set of RD signatures can be represented by $\mathrm{PC}_{1}$.

It can be informative to not only investigate how much of the variance that can be explained by $\mathrm{PC}_{s}$, but also how much of the standard deviation that can be explained by $\mathrm{PC}_{s}$. The same information is present in the variance and standard deviation, but since one is the square of the other, they emphasize different aspects of the information. This can be used to gain insight into whether the system behavior is linear or nonlinear. The standard deviation explained by $\mathrm{PC}_{s}$ is

$$
\gamma_{s}=\frac{\sqrt{\lambda_{s}}}{\sum_{s=1}^{p} \sqrt{\lambda_{s}}}
$$

Both $\eta_{s}$ and $\gamma_{s}$ have values between 0 and 1 , and $\sum_{s=1}^{p} \eta_{s}=\sum_{s=1}^{p} \gamma_{s}=1$.

A Pareto chart will be used to report the results of the PCA for both the variance and the standard deviation, see e.g. Figure $6 a$.

Since the RD signatures are calculated from an average of a finite number of signal segments, they will inherently include random error. This random error will prevent the first PC from explaining exactly $100 \%$ of the variance or standard deviation in practice. A threshold should therefore be formulated, expressing if all RD signatures differ enough from each other in the presence of nonlinearity indicators to accept the null hypothesis. To define such a threshold is not trivial, as several aspects influence the outcome of the analysis and is considered beyond the scope of the present study. Aspects such as, the number of RD signatures in the set, the number of lags in the RD signature, influence of the noise tail, eigenfrequency and decay rate of the system, noise contamination in the signal, sampling frequency. 
An empirical threshold is set in the present study for both the variance and the standard deviation explained by the first PC, to $\eta_{\text {thres }}=99.9 \%$ and $\gamma_{\text {thres }}=94 \%$ respectively. If $\eta_{1}<\eta_{\text {thres }}$ or $\gamma_{1}<\gamma_{\text {thres }}$ the null hypothesis is accepted, and the system behavior is interpreted as nonlinear. If $\eta_{1}>\eta_{\text {thres }}$ and $\gamma_{1}>\gamma_{\text {thres }}$ the null hypothesis is rejected and the system behavior is interpreted as linear. The thresholds presented here are to be used along with engineering judgment, as they are yet to be verified and validated.

\subsection{Parameterization of $R D$ signatures}

From the RD signatures, parameters can be calculated that describe certain properties of the set of RD signatures. Two parameters are presented, with the purpose of determining what aspect of each RD signature that differ from all the rest. Both parameters are visually presented similarly to the Modal Assurance Criterion (MAC), introduced by Allemang and Brown [28], where a value of 0 indicates that two mode shapes are completely different, and a value of 1 indicates that two modes shapes are completely the same.

For the purpose of detecting nonlinear behavior, a value of 1 indicates that the RD signatures are the same, and a value different from 1 means they differ to some degree.

Two aspects of the RD signatures are parameterized; the frequency, and the decay rate. Parameters that describe these aspects are presented in the following.

\subsubsection{Shape invariance criterion}

The Shape Invariance Criterion (SIC) was introduced by Asmussen [11, and is defined by

$$
S I C_{r, q}=\frac{\left|\hat{D}_{r} \hat{D}_{q}\right|^{2}}{\left|\hat{D}_{r}\right|^{2}\left|\hat{D}_{q}\right|^{2}}
$$

where $\hat{D}_{r}$ and $\hat{D}_{q}$ are two arbitrary RD signature estimates. The calculation performed is the same as for the MAC, where instead of comparing mode shapes, the RD signature estimates are compared. SIC was introduced as a method of investigating which triggering levels are appropriate to use in a specific signal. The SIC is sensitive to phase differences between the RD signatures, corresponding to a difference in frequency.

Results of the SIC analysis is presented in a similar way as the MAC matrix. However, instead of a mode index, an identifier to the TC that resulted in the RD signature is reported.

The SIC has a limitation in the fact that it is insensitive to changes in decay rate of the RD signatures. $\mathrm{SIC}$ is however very sensitive to frequency differences, and slight deviations from unity should be taken as an indicator of a nonlinearity.

Evaluating the results of the SIC analysis can be used to characterize the nonlinearity in question. Since SIC is not sensitive to changes in the decay rate or scaling, the analysis can be used to characterize a nonlinearity as being related to frequency.

\subsubsection{Relative area invariance criterion}

To address the limitation of the SIC not being sensitive to changes in the decay rate, a second criterion is introduced in the present study. The area of the squared RD signature is sensitive to the decay rate. We therefore define a parameter by first calculating the area of the squared RD signature by

$$
I=\int_{-\infty}^{\infty} \hat{D}_{y, y}^{2}(\tau) d \tau
$$

After the area of the square of all RD signatures are calculated, the relation between each area, and all others, are determined, and the relative area invariance criterion (RAIC) is defined as

$$
R A I C_{r, q}=\frac{I_{r}}{I_{q}}
$$


This relation is calculated for all pairs of areas, and set up in a RAIC matrix, similar to a MAC matrix. Finally these relations are normalized, such that the largest relation is 1, making it easy to compare with the SIC.

Results from the RAIC analysis is presented in the same way as the SIC, described above. Our experience show that RAIC is not as sensitive as SIC and deviations from unity should be significant before it indicates the presence of a nonlinearity.

Similarly to the SIC analysis, RAIC can be used to characterize the nonlinearity in question because it is insensitive to a difference in frequency. If there are some values in the RAIC matrix, significantly deviating from unity, this is an indicator of a nonlinearity related to the decay rate.

\section{Analysis procedure}

The combined steps that together makes up the novel procedure of detecting and characterizing nonlinear behavior of an SDOF system is described in this section. This analysis procedure is part of the NORD framework [20, 21, which is a strategy for applying the RD technique on nonlinear system to robustly estimate modal parameters.

A block diagram illustrating the procedure is presented in Figure 2, The diagram has blocks of four different patterns and colors, each describing an analysis step. The blue striped block represents the recorded vibration signal, and the derivative of the vibration signal. The red squared blocks describe the steps related to calculating the RD signatures. The green dotted blocks show the analysis of the RD signatures, and the black blocks show the evaluation of the analysis including the overall outcome of the procedure.

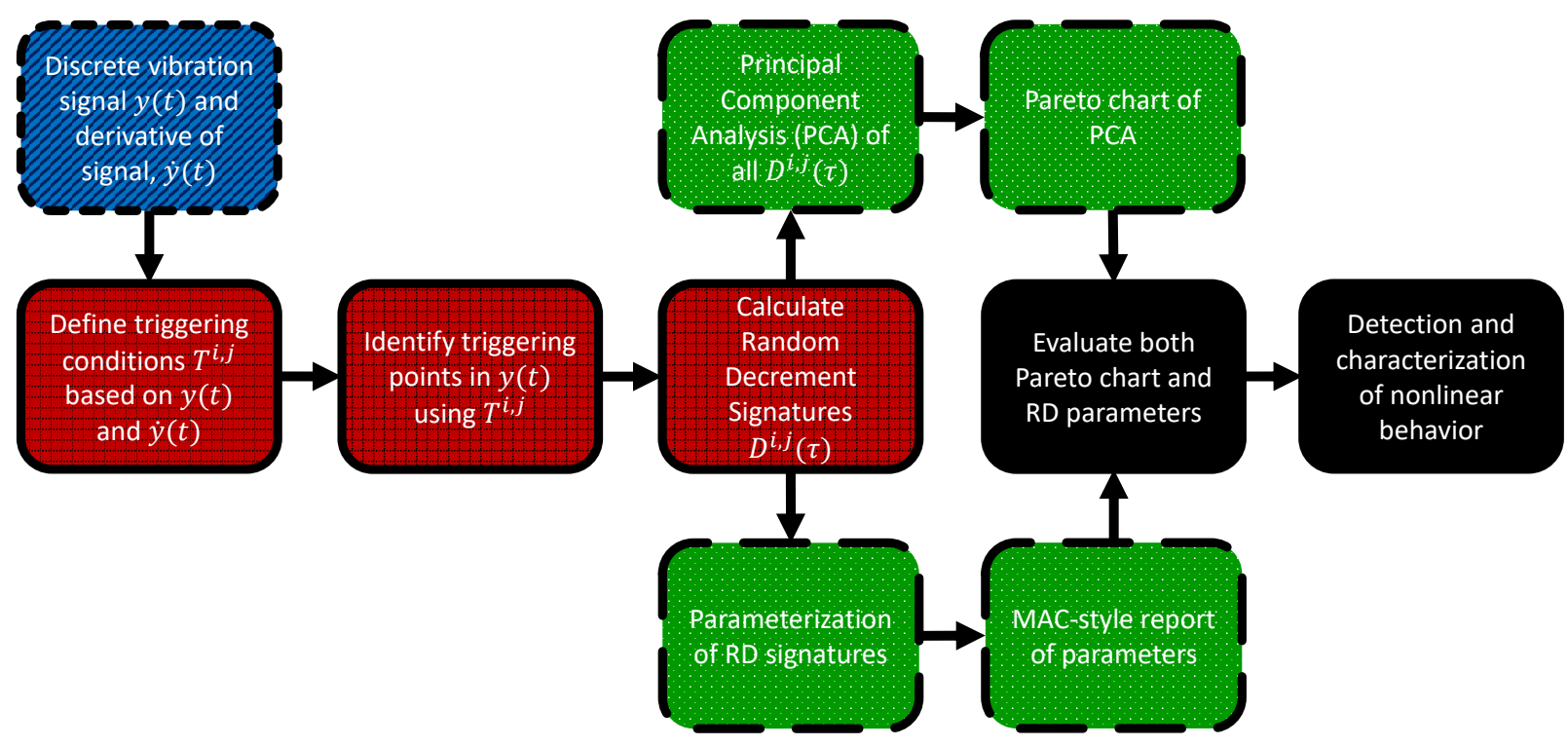

Figure 2: Novel analysis procedure within the NORD framework for detection and characterization of nonlinear behavior in SDOF systems under random force excitation.

From the response signal and the time derivative of the response signal in the blue block, the TCs are defined. In the leftmost red block, the parameters $a_{i}$ and $b_{j}$ are calculated and used to define $T^{i, j}$ as in Equation (15). Parameters $a_{i}$ and $b_{j}$ are defined generally as a function of the standard deviation of the response signal and derived signal and presented in tables 1 and 2 . These parameters are calculated for 
the specific response signal under investigation, and will be unique for each test case. In total there are $(m-1) \times(n-1)=(7-1) \times(5-1)=24 \mathrm{TCs}$, each representing a unique initial condition.

With this definition of the parameters $a_{i}$ and $b_{j}$, it is impossible for any time sample to be identified as TP in more than one $T^{i, j}$, meaning all TPs used in the analysis are unique. This is the case because there are no overlapping between the amplitude bands defined by $a_{i}$.

The number of TPs is of some importance, since it determines the number of signal segments being averaged in Equation (8). A study by Chang 29] recommends 1000 or 2000 TPs for estimating frequency or damping respectively. Length of the response time series, and the parameters $a_{i}$, and $b_{j}$, have been chosen to accommodate the recommendation of 1000 TPs. The lower number is chosen because no parameter estimation is to be performed on the $\mathrm{RD}$ signatures in the present procedure.

Next step in the procedure is to apply $T^{i, j}$ to the response signal and thereby identify TPs. This is performed in the second red block from the left.

Once the TPs have been obtained, the RD signatures $D^{i, j}$ are calculated, as represented by the third red block from the left. The block size of all RD signatures calculated in the present study is 2049. This block size is chosen based on an empirical analysis where a few different values were investigated. If the block size is too small, the parameterization and PCA will not yield a useful result because the RD signatures will appear to be equal. A too large block size comes with a computational cost, and no added information. The analyst performing this operation therefore has to test a few different block sizes to see which is best. Here a block size representing 2 seconds of time history was appropriate.

The two steps of first identifying TPs and then calculating RD signatures are repeated, but this time with the negative of the response signal $-y(t)$. This is done to reveal information about how the positive response behaves compared to the negative response. This is expected to be relevant for systems with boundary condition nonlinearities, or cracks opening and closing for positive and negative response respectively. It is not expected to have an effect of the outcome in the test cases investigated in the present study, but is included for completeness. The total number of RD signatures being calculated every time the analysis procedure is applied, is therefore twice the number of TCs, resulting in 48 .

As mentioned in Section 2.3 the RD signatures are normalized to z-score before performing the PCA. This normalization scales the RD signatures differently if they are not equal, which is useful for visual inspection, which is the first step in interpreting the results.

\begin{tabular}{|c|c|c|}
\hline $\begin{array}{c}\text { Index } \\
\boldsymbol{i}\end{array}$ & $\begin{array}{c}\text { Amplitude } \\
\boldsymbol{a}_{\boldsymbol{i}} \boldsymbol{\sigma}_{\boldsymbol{y}}\end{array}$ & $\begin{array}{c}\text { Amplitude range } \\
{\left[\boldsymbol{a}_{\boldsymbol{i}}-\boldsymbol{a}_{\boldsymbol{i}+\mathbf{1}}\right] / \boldsymbol{\sigma}_{\boldsymbol{y}}}\end{array}$ \\
\hline 1 & 0.75 & {$[0.75-0.99]$} \\
\hline 2 & 0.99 & {$[0.99-1.23]$} \\
\hline 3 & 1.23 & {$[1.23-1.48]$} \\
\hline 4 & 1.48 & {$[1.48-1.72]$} \\
\hline 5 & 1.72 & {$[1.72-1.96]$} \\
\hline 6 & 1.96 & {$[1.96-2.20]$} \\
\hline 7 & 2.20 & - \\
\hline
\end{tabular}

\begin{tabular}{|c|c|c|}
\hline $\begin{array}{c}\text { Index } \\
\boldsymbol{j}\end{array}$ & $\begin{array}{c}\text { Slope } \\
\boldsymbol{b}_{\boldsymbol{j}} / \boldsymbol{\sigma}_{\dot{\boldsymbol{y}}}\end{array}$ & $\begin{array}{c}\text { Slope range } \\
{\left[\boldsymbol{b}_{\boldsymbol{j}}-\boldsymbol{b}_{\boldsymbol{j}+\mathbf{1}}\right] / \boldsymbol{\sigma}_{\boldsymbol{\dot { y }}}}\end{array}$ \\
\hline 1 & 0 & {$[0-0.5]$} \\
\hline 2 & 0.5 & {$[0.5-1]$} \\
\hline 3 & 1 & {$[1-1.5]$} \\
\hline 4 & 1.5 & {$[1.5-2]$} \\
\hline 5 & 2 & - \\
\hline
\end{tabular}

Table 1: Triggering parameter $a_{i}$ scaled to std. dev. of Table 2: Triggering parameter $b_{j}$ scaled to std. dev. of time derivaresponse signal $y(t)$. tive of response signal $\sigma_{\dot{y}}$.

\section{Numerical case study}

A numerical case study is performed by analyzing the response of a SDOF system with a stick slip friction nonlinearity, illustrated in Figure 3. The nonlinearity is implemented using a Jenkins element [30], consisting of a Coulomb friction $F_{d}$ and a spring $k_{d}$.

The mechanical parameters describing the linear part of the system are mass $m=0.23 \mathrm{~kg}$, stiffness $k=$ $1650 \mathrm{~N} / \mathrm{m}$, and damping $c=0.13 \mathrm{~N} /(\mathrm{m} / \mathrm{s})$. The stiffness in the Jenkins element is given as $k_{d}=1900 \mathrm{~N} / \mathrm{m}$, and is referred to as the backing stiffness. The friction force $F_{d}$ is varied between tests in order to investigate the analysis procedure with different degrees of nonlinearity. A zero mean, stationary, Gaussian signal with 


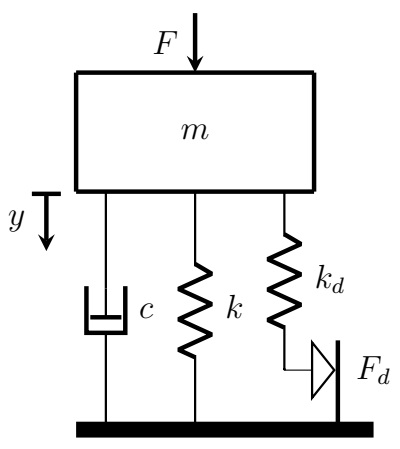

Figure 3: Single degree of freedom system with stick-slip friction implemented as a Jenkins element.

a standard deviation of $5 \mathrm{~N}$ is used as the excitation force $F(t)$ for all test cases. The motion of a SDOF system with a Jenkins element is governed by

$$
m \ddot{y}(t)+c \dot{y}(t)+k y(t)+f_{n l}=F(t), \text { where } f_{n l}= \begin{cases}k_{d} y, \text { for }|y|<F_{d} / k_{d} \\ F_{d}, \text { for }|y| \geq F_{d} / k_{d}\end{cases}
$$

The nonlinear force $f_{n l}$ is determined by the relationship between $|y|$ and $F_{d} / k_{d}$. The numerical stick slip system investigated in the present study is explained here by describing its behavior with four general friction forces $F_{d}$.

For a very low $F_{d},|y| \gg F_{d} / k_{d}$. Here, an insignificant amount of energy is dissipated because of $F_{d}$ and the only relevant damping force in the system is $c \dot{y}$. In this case the backing stiffness $k_{d}$ is compressed with an insignificant amount, and the total stiffness of the system is $k$. This is the slip phase, where the response of the system appears unaffected by the Jenkins element.

A low $F_{d}$ results in $|y|>F_{d} / k_{d}$. In this situation, a significant amount of the total energy dissipation is caused by $F_{d}$ and the relative damping of the system is greater than the linear damping caused by $c \dot{y}$. The total stiffness of the system is affected by $k_{d}$. With a low $F_{d}$, only a slight increase in total stiffness is observed. This is a stick/slip phase.

For an intermediate $F_{d}$, the friction has a severely nonlinear effect on the response of the system. Here $|y| \approx F_{d} / k_{d}$, and the energy dissipation is dominated by the friction force $F_{d}$, and the relative damping becomes extremely high. In addition, the restoring force from the backing stiffness has a large influence on the total stiffness, and the overall restoring force is significantly larger than $k y$. This is also a stick/slip phase.

For a high $F_{d}$, the restoring force produced by the displacement response and the backing stiffness $y \cdot k_{d}$, is not enough to overcome $F_{d}$. In this case the Jenkins element behaves purely as a linear stiffness, and the total stiffness of the system is $k+k_{d}$. This is known as the stick phase.

It should be noticed that the system is excited by a random force, and therefore has a random response. This means that the system will have a displacement response magnitude that causes the system to gradually shift between the stick phase, the stick/slip phase, and the slip phase as the structure vibrates. Overall, this means that the behavior of this system is nonlinear to varying degrees, and involves both amplitude dependent damping and amplitude dependent frequency. This makes the system very suitable to investigate the detection and characterization method proposed in the present study.

Based on this description of the system, linear behavior is assumed for very low $F_{d}$ and high $F_{d}$. Varying degree of nonlinear behavior involving both frequency and damping is expected for all other cases.

The forced response of the system is simulated using a digital filter based method, presented by Ahlin et al. 31. A sampling frequency $f_{s}=2048 \mathrm{~Hz}$, and a time history length of $600 \mathrm{~s}$ is used in the simulation of all test cases.

The 6 cases are investigated, and presented in table 3 The friction force $F_{d}$ and $\sigma_{y}$ in each particular case, and the ratio $\frac{F_{d}}{\sigma_{y} k_{d}}$ that is used to estimate the friction force level, are presented in the table. 


\begin{tabular}{|c|c|c|c|c|}
\hline $\begin{array}{c}\text { Case } \\
\#\end{array}$ & $\begin{array}{c}\text { Friction force } \\
\boldsymbol{F}_{\boldsymbol{d}}[\mathrm{N}]\end{array}$ & $\begin{array}{c}\text { Signal std. dev. } \\
\boldsymbol{\sigma}_{\boldsymbol{y}}[\mathrm{m}]\end{array}$ & $\begin{array}{c}\text { Force ratio } \\
\frac{\boldsymbol{F}_{\boldsymbol{d}}}{\boldsymbol{\sigma}_{\boldsymbol{y}}}[-]\end{array}$ & $\begin{array}{c}\text { Estimated } \\
\text { friction level }\end{array}$ \\
\hline 1 & 0.01 & 0.0053 & 0.001 & very low \\
\hline 2 & 0.06 & 0.0040 & 0.008 & low \\
\hline 3 & 0.32 & 0.0014 & 0.126 & intermediate \\
\hline 4 & 0.77 & 0.0008 & 0.501 & intermediate \\
\hline 5 & 1.84 & 0.0008 & 1.189 & intermediate \\
\hline 6 & 25 & 0.0034 & 3.861 & high \\
\hline
\end{tabular}

Table 3: Presentation of the friction in numerical test cases. The estimated friction force level is based on results of an analysis of the same dataset presented by Vesterholm et al. [18.

\subsection{Results of numerical case}

Results for the numerical cases of various friction forces $F_{d}$ are presented in this section.

As mentioned in section 3 , the aim is to achieve $N=1000$ TPs when calculating each individual RD signature. For the entire analysis of all numerical cases, the lowest number of TPs for any RD signature was $N=887$, and the recommendation is considered followed to a reasonable degree.

Results of the numerical test cases are separated in two groups, intermediate friction level, and the rest. This is done for easy comparison of systems expected to exhibit severe nonlinear behavior (intermediate), and systems expected to exhibit little nonlinear behavior (the rest). The set of z-score normalized RD signatures $D^{i, j}$ are presented for test cases 1, 2 and 6 in Figure 4 , cases 3, 4 and 5 in Figure 5 , The indices $i$ and $j$ refers to the parameters $a_{i}$ and $b_{j}$ that are used in the specific TC $T^{i, j}$ from Equation (15). In total, there are 24 TCs. Since the RD calculations are performed on both the measured signal, and the negative of the measured signal, there are 48 individual RD signatures in each plot. The individual $D^{i, j}$ resulting from $T^{i, j}$ is identified by the color of its line, specified in the colorbar. From the figures illustrating the RD signatures, the reader should get a general impression of how the $48 \mathrm{RD}$ signatures behave in relation to each other. The plots are not meant to illustrate how each specific $D^{i, j}(\tau)$ behaves.

From a visual inspection of the z-score normalized RD signatures in Figure 4 a, no apparent difference is seen for the various TCs.

With a low friction force, the RD signatures in Figure $4 \mathrm{~b}$ indicate a difference in behavior with different TC. A slight difference in peak amplitude is visible. Starting from $T^{1,1}$ moving up the colorbar to $T^{6,4}$ the peak amplitude is increasing slightly. When the friction force is high, as in Figure $4 \mathrm{c}$, the RD signatures all overlap, and no difference between them can be seen from a visual inspection.

For the three cases with an intermediate friction force illustrated in Figure 5 , the difference in behavior of the RD signatures are very clear.

The analysis procedure described in section 3 is applied on the numerical test cases. The outcome of the analysis is grouped the same way as the plots for the RD signatures above for easy comparison, and is presented in Figures 6 and 7. The figures illustrate the PCA in the form of Pareto charts for variance and standard deviation, and the parameters SIC and RAIC are illustrated in MAC style plots. The captions specify the case number, and the level of friction force for the specific test case according to Table 3 .

The Pareto charts, which are the two leftmost columns of panes, in Figures 6 and 7 illustrate two things. First, they show the value of variance or standard deviation of the first 5 PCs with a bar chart. Second, they show with a percentage, how much of the total variance and standard deviation, respectively that can be explained by each of the first 5 PCs in a cumulative way.

It is clear that the variance explained by $\mathrm{PC}_{1}$ is near $100 \%$ for all 6 test cases. Only cases 4 and 5 show a slight drop to approximately $97 \%$. This small difference between cases makes it difficult to evaluate whether the system behavior is linear or nonlinear, based on this analysis parameter alone.

When examining the Pareto charts for the standard deviation, a significant variation between cases is observed. For cases 1,2, and 6; the standard deviation explained by $\mathrm{PC}_{1}$ is above $94 \%$. The $\mathrm{PC}_{1}$ in the remaining cases 3,4 , and $5,78.08 \%$ or less, indicating they exhibit nonlinear behavior. This means, a clear distinction between cases with an intermediate friction force level, and the other cases can be made using this parameter. Going into detail of cases 1,2 , and 6 , it can be seen that in cases 1 and $6, \mathrm{PC}_{1}$ explains 


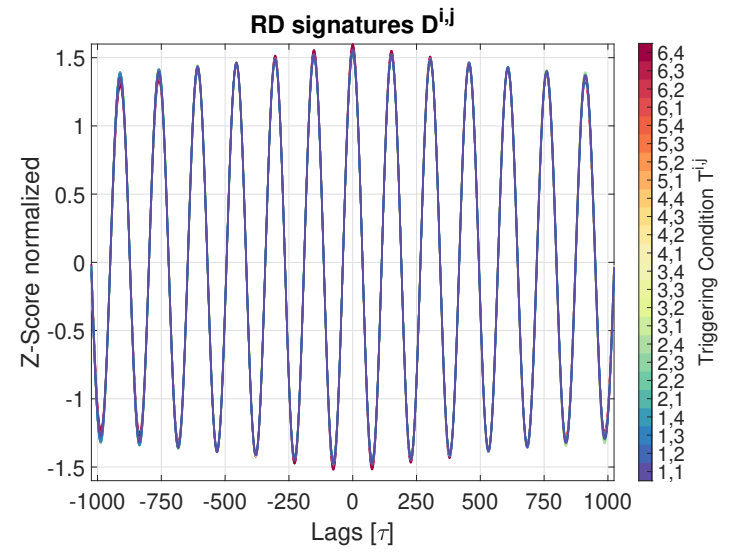

(a) Case 1, very low friction force level.

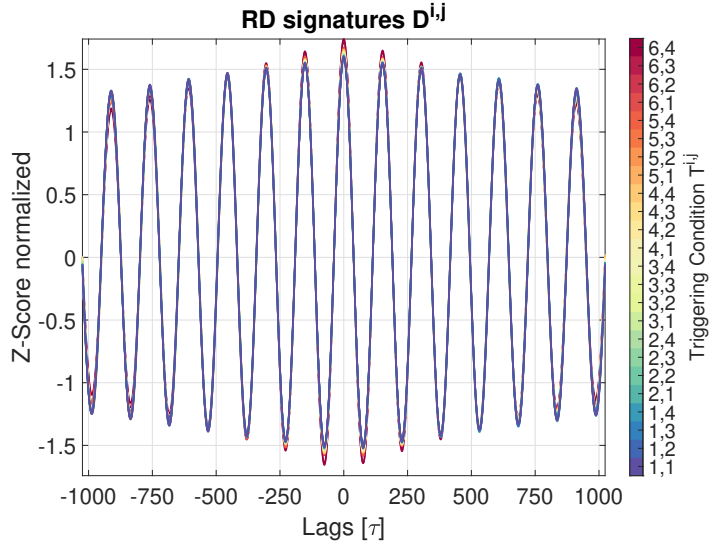

(b) Case 2, low friction force level.

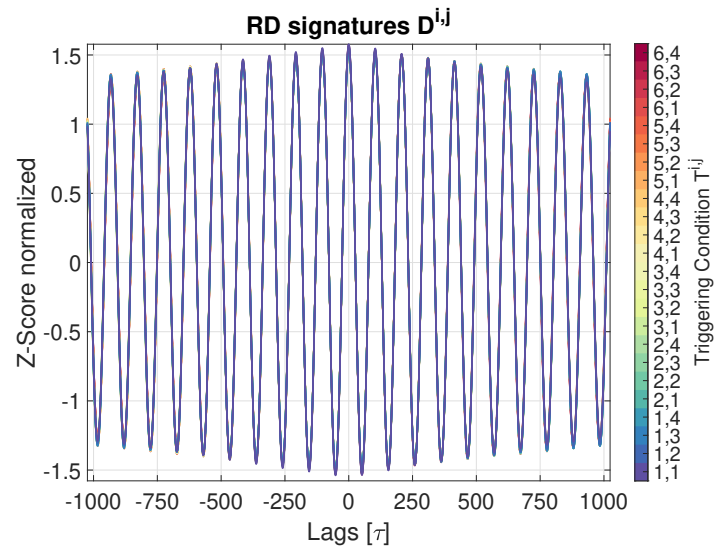

(c) Case 6 high friction force level.

Figure 4: Random decrement signatures for numerical test cases 1, 2 and 6, as described in table 3 Colorbar specify indices for the parameters in TC $T^{i, j}$ applied to calculate corresponding RD signature. If all RD signatures overlap - it is an indication of linear behavior, as seen for cases 1 and 6 . It not all RD signatures overlap, it is an indication of nonlinear behavior.

around $96 \%$ of the standard deviation, while $94.1 \%$ is explained by $\mathrm{PC}_{1}$ in case 2 . This could indicate that case 2 has a slightly nonlinear behavior. This observation is consistent with the fact that only cases 1 and 6 should exhibit linear behavior.

The two parameters SIC and RAIC are illustrated in the two rightmost column of panes in Figures 6 and 7. The $\mathrm{x}$ - and y axes show the indices for the RD signature resulting from TC $T^{i, j}$. The resulting SIC and RAIC values, found using Equations (23) and 25) respectively, are represented by the color specified in the color bar.

Comparing all SIC plots between Figures 6 and 7. cases 1, 2, and 6 stand out, because all SIC values here are unity. SIC is a measure of the frequency relationship is between pairs of RD signatures. This means the analysis procedure revealed no difference in frequency for all TCs investigated for the cases 1,2 , and 6 . For the remaining cases 3, 4, and 5, all with intermediate friction force level, SIC values drop below unity. In case 3, SIC drops only slightly below unity, where for cases 4, and 5 SIC values are between 1 and 0.66 . This reflects that case 3 has a slight shift in frequency for the different TCs investigated. Cases 4 and 5 have significant shifts in frequency for different TCs.

The RAIC parameter appears to have some amount of random variation, and deviations from unity is not interpreted as strictly as for SIC. Including this consideration, the RAIC for cases 1 and 6 are similar, with values between 1 and 0.85 randomly distributed in the plot. The RAIC plot for case 2 shows a slight trend with the highest values of 1 in the lower left corner and dropping as a global trend towards the upper 


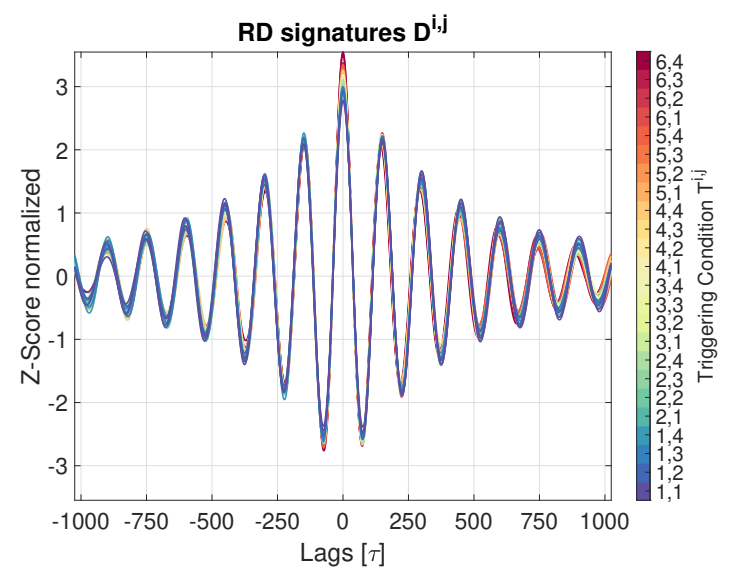

(a) Case 3, intermediate friction force level.

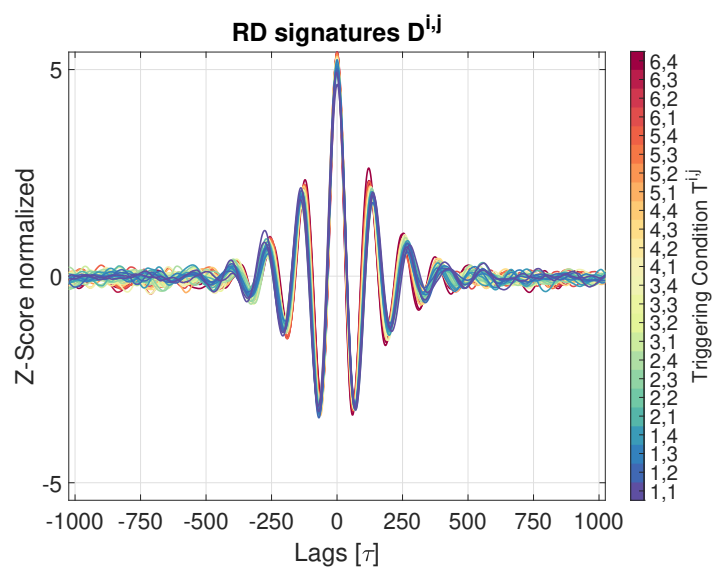

(b) Case 4 intermediate friction force level.

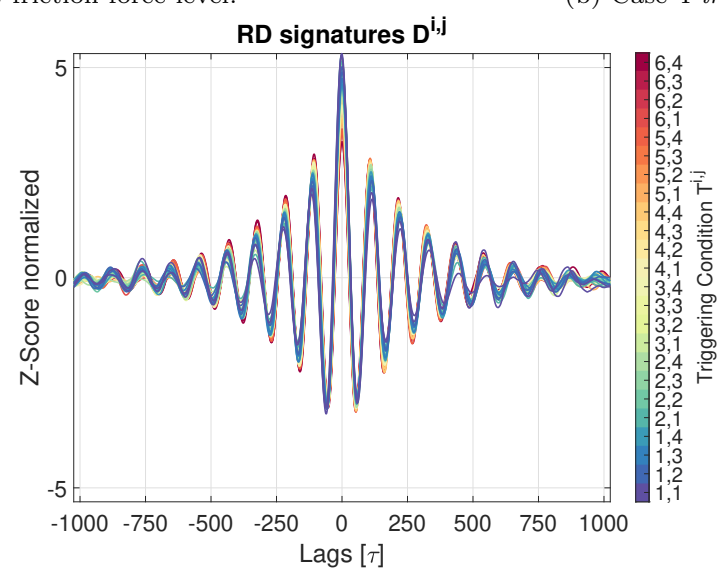

(c) Case 5 intermediate friction force level.

Figure 5: Random decrement signatures for numerical test cases 3,4,5, as described in table 3 Colorbar specify indices for the parameters in TC $T^{i, j}$ applied to calculate corresponding RD signature. Since not all RD signatures overlap, it is an indication of nonlinear behavior.
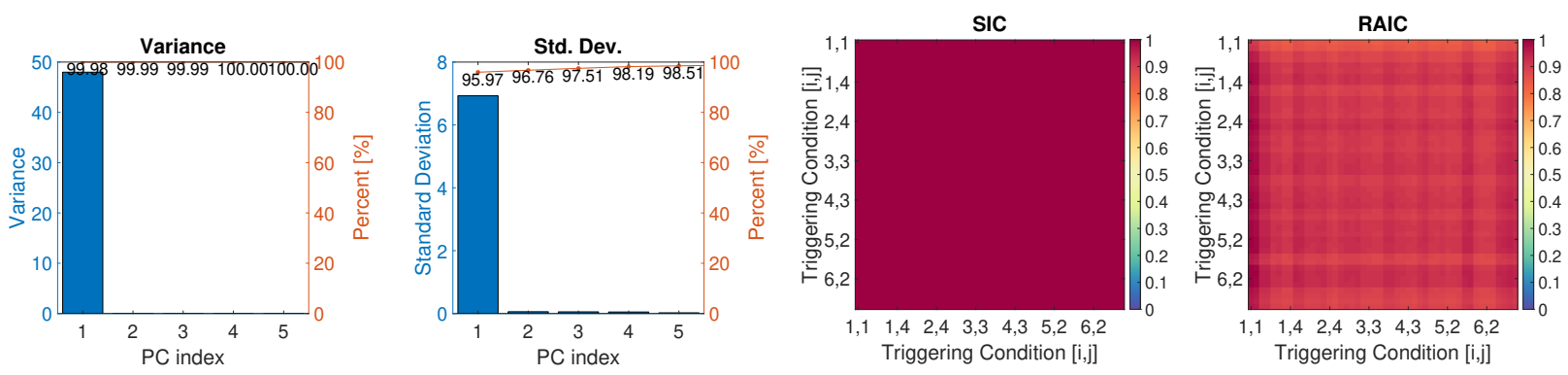

(a) Case 1, very low friction force level.

right corner with a minimum value of 0.68 . This indicates that $D^{6,4}$ has the largest area, and $D^{1,1}$ has the smallest. A local trend is also present for each triggering level, which are the $i$ indices. Considering only $i=1$ for case 2 , a local trend appears where a greater index $j$ results in a greater RAIC. This local trend can also be observed for other triggering levels in case 2. Observing both the local and global trends means that $T^{i, j}$ with high triggering level and large slope results in a larger area than for low triggering level and small slope. A trend similar to, and more pronounced than case 2 is observed in case 3 . No trends are observed in RAIC for case 4, but values drop significantly below unity to indicate a difference in area between the RD 

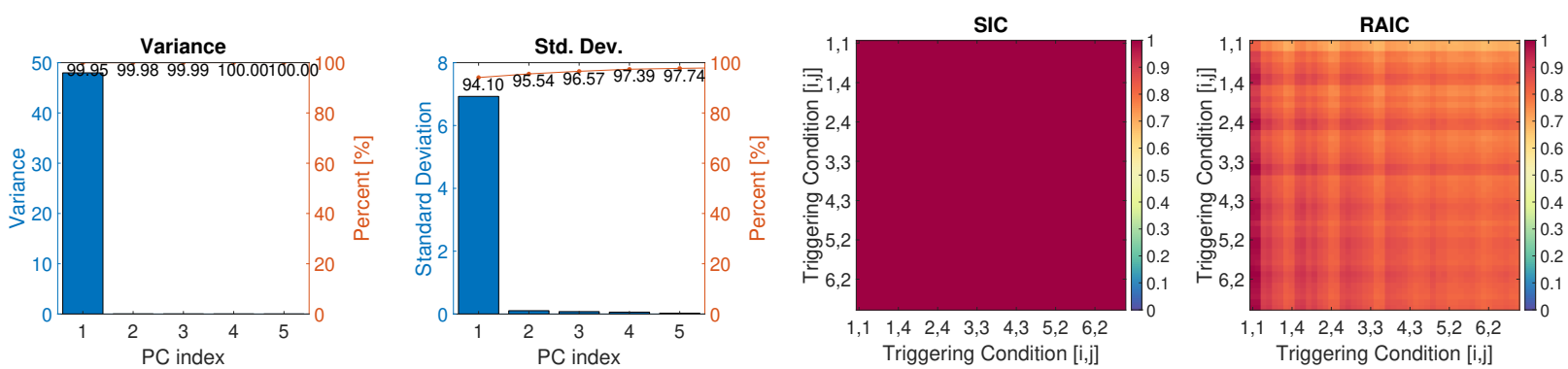

(b) Case 2, low friction force level.
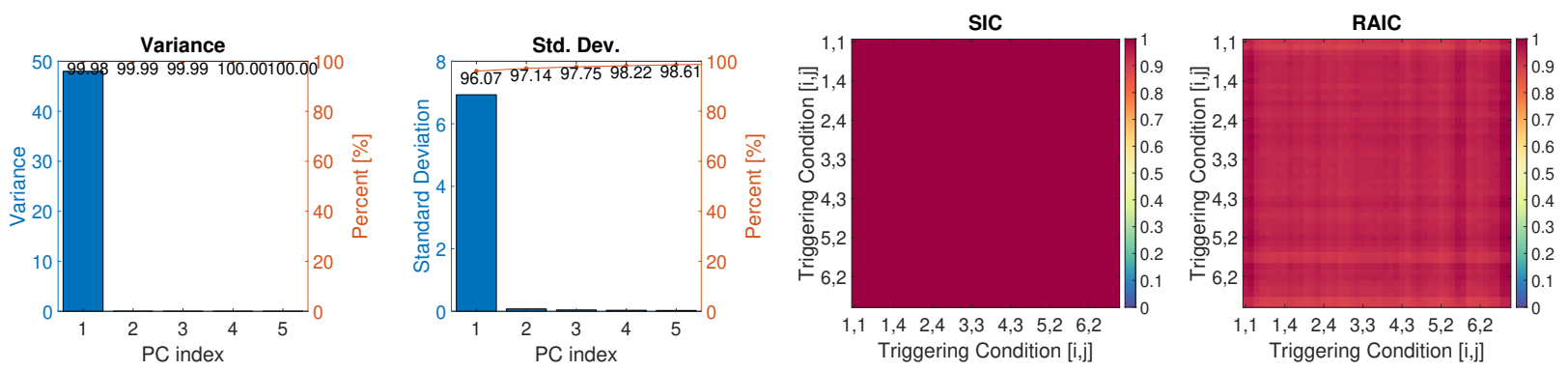

(c) Case 6, high friction force level.

Figure 6: Outcome of analysis procedure in Figure 2 applied to numerical test cases 1, 2 and 6 presented in Table 3 one row of plots per test case. From the left, Pareto charts for variance and standard deviation are interpreted by comparing $\mathrm{PC}_{1}$ with the thresholds defined in section 2.3 to be $\eta_{\text {thres }}=99.9 \%$ and $\gamma_{\text {thres }}=94 \%$ for the variance and standard deviation respectively. Continuing to the left SIC and RAIC summarize the parameterization of RD signatures. For both SIC and RAIC, a value less than 1 corresponds to a pair of RD signatures being dissimilar. Values in the SIC or RAIC plots that are significantly less than 1 are interpreted as nonlinear behavior related to frequency or damping respectively.
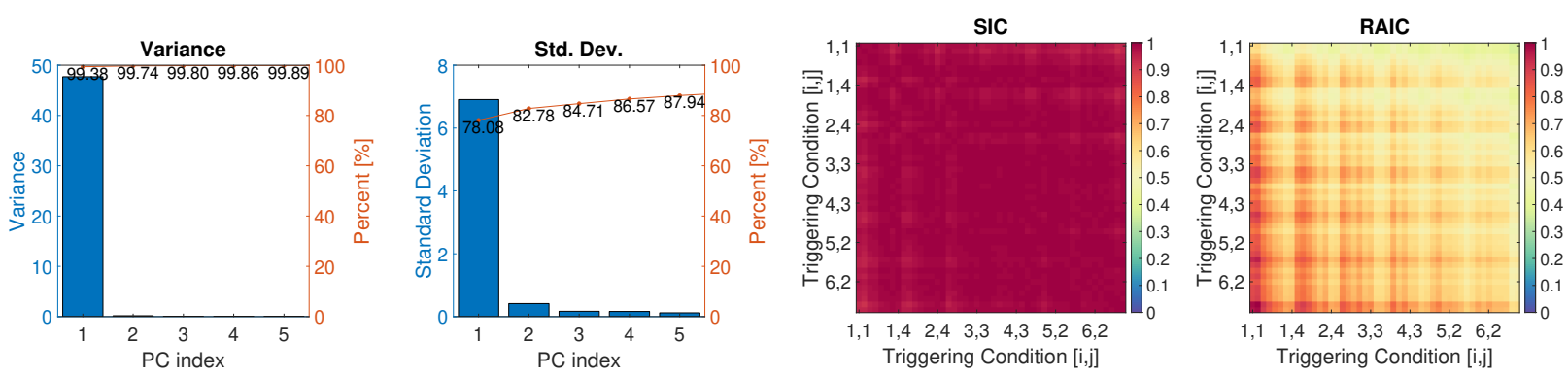

(a) Case 3, intermediate friction force level.
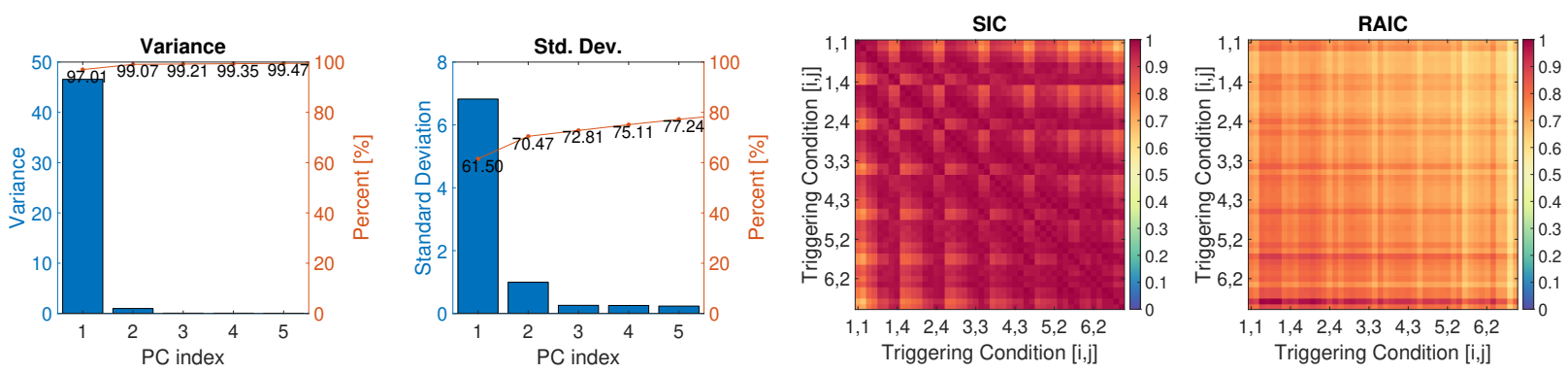

(b) Case 4, intermediate friction force level.

signatures. Case 5 shows a clear trend in RAIC, both globally and locally. The trends are very pronounced compared to cases 2 and 3, but has a different characteristics, meaning $T^{i, j}$ with low triggering level and 

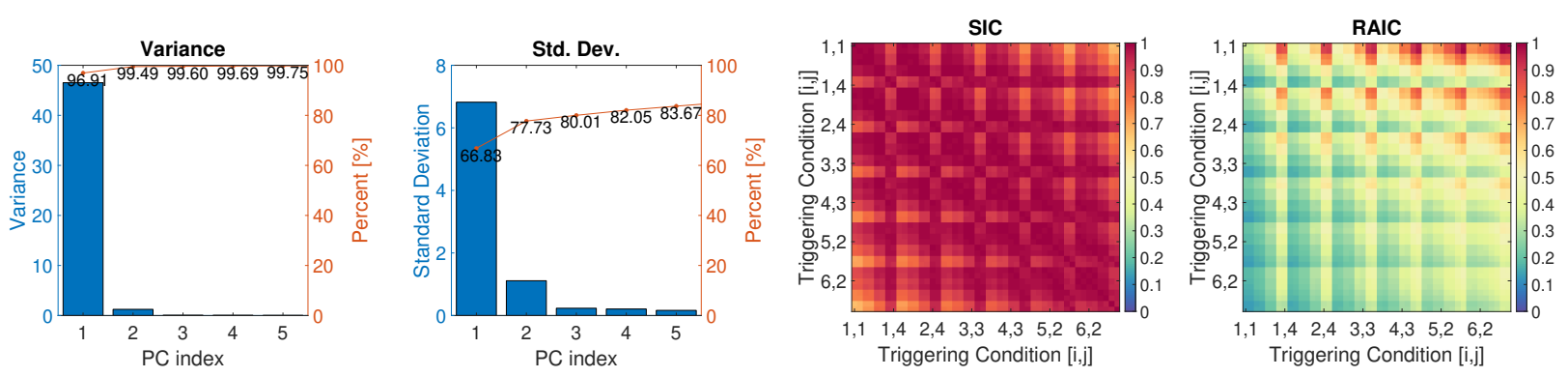

(c) Case 5, intermediate friction force level.

Figure 7: Outcome of analysis procedure in Figure 2 applied to numerical test cases 1-6 described in Table 3 one row of plots per test case. From the left, Pareto charts for variance and standard deviation are interpreted by comparing $\mathrm{PC}_{1}$ with the thresholds defined in section 2.3 to be $\eta_{\text {thres }}=99.9 \%$ and $\gamma_{\text {thres }}=94 \%$ for the variance and standard deviation respectively. Continuing to the left SIC and RAIC summarize the parameterization of RD signatures. For both SIC and RAIC, a value less than 1 corresponds to a pair of RD signatures being dissimilar. Values in the SIC or RAIC plots that are significantly less than 1 are interpreted as nonlinear behavior related to frequency or damping respectively.

large slope results in a larger area than for high triggering level and small slope.

\subsubsection{Outcome of analysis of numerical case}

Cases 1 and 6, with very low and high friction force level respectively, can both be concluded to have linear behavior based on the analysis performed. There were no parameters indicating that any of the TCs investigated, resulted in a RD signature with a frequency or damping different from the others. Therefore the alternative hypothesis has to be accepted, and the system behavior is interpreted as being linear. This is consistent with the expected behavior of the system.

In case 2 , the standard deviation explained by $\mathrm{PC}_{1}$ was slightly lower than for cases 1 and 6 , and very close to the threshold of $94 \%$ which could indicate nonlinear behavior. The SICs were all unity and indicated no nonlinear behavior regarding frequency. The RAIC revealed a slight trend meaning there is nonlinear behavior related to the damping. In conclusion, case 2 is evaluated to exhibit slightly nonlinear behavior only related to damping.

Case 3 has a clear nonlinear behavior, based on 3 of the 4 examined parameters. The standard deviation explained by $\mathrm{PC}_{1}$ is significantly below $100 \%$, meaning nonlinear behavior can be detected. The slight deviation from unity in SIC indicates that there is a slight nonlinearity related to frequency. The RAIC parameter shows that the nonlinearity is related to damping, as values drop below 0.4 .

In case 4 all four parameters indicate that it exhibits nonlinear behavior. Standard deviation explained drops significantly below $100 \%$ meaning nonlinear behavior can be detected. Both SIC and RAIC has values significantly below unity, and the nonlinear behavior can be characterized as related to both frequency and damping.

Case 5 is concluded to exhibit nonlinear behavior. Standard deviation explained by $\mathrm{PC}_{1}$ is low, meaning that nonlinear behavior is detected. A SIC with values significantly below unity indicates the nonlinear behavior is characterized as related to frequency. In addition, RAIC values significantly below unity characterizes the nonlinear behavior as also being damping related.

\section{Experimental case study}

An experimental case study was designed to behave approximately as a SDOF stick slip system. The experimental data obtained from the setup has previously been analyzed by Vesterholm et al. [18, with the purpose of investigating the RD technique as a method of obtaining the amplitude dependent modal parameters of a stick-slip system.

The experimental setup is pictured in Figures $8 \mathrm{a}$ and $8 \mathrm{~b}$. A steel base is clamped to a vibration isolated workbench, with a $120 \mathrm{~mm}$ long M4 threaded rod bolted to it. A $30 \mathrm{~mm} \times 30 \mathrm{~mm} \times 30 \mathrm{~mm}$ steel cube 
is bolted to the other end of the threaded rod. The fixed rod and steel cube form an approximately linear single-degree-of-freedom system in the direction of the accelerometer. A stick slip mechanism is added to the system in the form of a spring and a friction mechanism. The spring consists of an additional M4 threaded rod of $128 \mathrm{~mm}$ length. The friction mechanism is a $16.5 \mathrm{~mm}$ diameter cylinder made from black polyoxymethylene (POM) in contact with a felt disc glued to the steel cube. The threaded rod is screwed into the POM cylinder in one end and clamped to a lab fixture above the steel cube in the other end such that it is possible to turn the rod. The POM cylinder is pushed into the felt disc with various distances to the steel controlled by turning the rod. Turning the threaded rod changes its length up to $0.83 \mathrm{~mm}$ between tests. This changes its stiffness, but the amount is considered insignificant.

As a comparison with the conceptual drawing in Figure 3 the lower rod and the steel cube in Figure 8 a constitutes the linear mechanical parameters $m, c$ and $k$. The mass is primarily located in the steel cube, the stiffness is from the bending of the rod, material damping in the rod and the bolted joints are assumed to behave as viscous damping. The friction connection between the POM and felt is the friction force $F_{d}$. The stiffness of the upper rod is the backing stiffness $k_{d}$. In total this system has a behavior that approximates a single-degree-of-freedom stick slip system.

To imitate ambient or natural excitation, the structure is excited by brushing the steel cube with a paint brush which is seen in the bottom of Figure 8a. It is attempted to excite with a stationary random force, but since this is done by the experimentalist some variation is expected.

The vibration of the system is measured with a wax mounted uniaxial accelerometer with a sampling frequency of $f_{s}=2048 \mathrm{~Hz}$. The measurement time is 600 seconds. The resonance frequency of the linear system consisting only of the lower rod and the steel cube is around $13 \mathrm{~Hz}$.

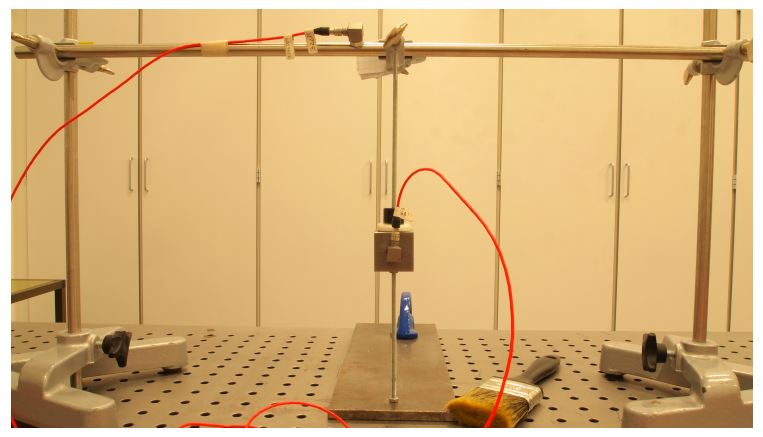

(a) Overview of experimental setup, including the brush used to excite the structure.

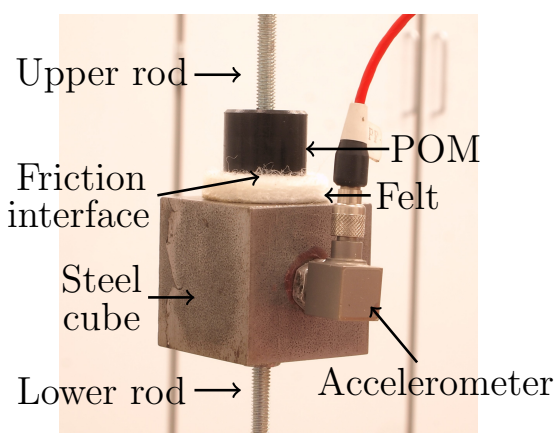

(b) Steel mass with felt glued on top, that has frictional contact with block of POM. Vibration is measured using uniaxial accelerometer.

Figure 8: Experimental setup.

6 different friction force levels are investigated in the present study. The friction force level is controlled by the gap size between the POM and the steel cube. With a large gap size, the POM has only a small degree of contact with the felt disc, and therefore a low friction force. A small gap results in high degree of contact, and therefore a high friction force. Since the friction force has not been measured it is unknown, and only the gap size is reported as a metric of the friction force level. The 6 gap sizes investigated are $\infty, 5.92,5.44,5.38,5.29$, and $4.96 \mathrm{~mm}$, measured with calipers. In Table 4 the test cases are presented along with an estimated friction force level. This estimation is made based on results from a different analysis of the same dataset by Vesterholm et at. [18. The first gap size of infinity means that no friction is present in the system, as the POM cylinder is not installed for this test, and the system is assumed to behave linearly.

\subsection{Results of experimental case}

Results of the experimental tests case are presented in this section. 


\begin{tabular}{|c|c|c|}
\hline $\begin{array}{c}\text { Case } \\
\#\end{array}$ & $\begin{array}{c}\text { Gap size } \\
{[\mathrm{mm}]}\end{array}$ & $\begin{array}{c}\text { Estimated } \\
\text { friction level }\end{array}$ \\
\hline 1 & $\infty$ & none \\
\hline 2 & 5.92 & low \\
\hline 3 & 5.44 & intermediate \\
\hline 4 & 5.38 & intermediate \\
\hline 5 & 5.29 & intermediate \\
\hline 6 & 4.96 & high \\
\hline
\end{tabular}

Table 4: Presentation of the friction in experimental test cases.

As mentioned in section 3, the aim is to achieve $N=1000 \mathrm{TPs}$ when calculating each individual RD signature. For the entire analysis of the experimental case, the lowest number of TPs for any RD signature was $N=971$, and the recommendation is considered followed to a reasonable degree.

Results of the experimental test cases are separated in two groups, intermediate friction level, and the rest. This is done for easy comparison of systems expected to exhibit severe nonlinear behavior (intermediate), and systems expected to exhibit little nonlinear behavior (the rest). In Figures 9 and 10 the z-score normalized $\mathrm{RD}$ signatures $D^{i, j}(\tau)$ are illustrated. Similar to the results of the numerical test case, indices $i$ and $j$ refer to the parameters $a_{i}$ and $b_{j}$ that are used in the specific TC $T^{i j}$ from Equation (15).

First a visual inspection of the RD signatures is presented, then results of the analysis procedure, and finally the evaluation and outcome are presented for each experimental test case.

The graphs are of the same format as in the numerical test case, and for the explanation of how to read the graphs, the reader is referred to Section 4.1 .

From a visual inspection of Figure 9a with case 1 where no friction is present in the system, a slight difference in decay between $\mathrm{RD}$ signatures is present in the negative lags.

For case 2 with a low friction force level in Figure $9 \mathrm{~b}$ a difference between the RD signatures is only just observable, and very small for all lags.

Case 6 with an estimated high friction force level in Figure $9 \mathrm{c}$ a minor difference between the RD signatures is observed in the negative lags.

Cases 3, 4, and 5 have intermediate friction force level, and in Figure 10 a very significant difference between RD signatures is observed. The analysis procedure described in Figure 2 is applied on all experimental test cases, and the Pareto charts of the variance and standard deviation along with the SIC and RAIC are presented in Figures 11 and 12.

When comparing the Pareto charts in Figures 11 and 12 for variance explained by $\mathrm{PC}_{1}$ for all experimental test cases, a trend similar to the numerical test case is observed; all are very close to $100 \%$. Only cases 3,4 , and 5 drop below $99 \%$. This small difference between cases makes it difficult to extract information about linearity of the behavior based on this parameter.

The Pareto charts for standard deviation explained by $\mathrm{PC}_{1}$ has larger dynamics between test cases, meaning difference in behavior of the test cases can be differentiated by observing this parameter.

SIC for test cases 1 and 2 are very similar, and deviating only slightly from unity, minimum value for cases 1 and 2 are 0.99. This is considered an insignificant deviation. Case 6 has a minimum SIC value of 0.93 , a large enough deviation to be considered significant. In cases 3,4 , and 5 , SIC is deviating significantly from unity with minimum values of $0.87,0.43$, and 0.77 respectively, indicating a difference in frequency between the individual RD signatures in each case.

The RAICs for cases 1 and 2 have very similar minimum values, 0.77 and 0.84 respectively. It is noteworthy that in case 1, which has no friction, a slight trend is observed in the RAIC plot. The TCs with low $i$ indices result in a relatively lower area than TCs with high $i$ indices. The opposite trend is observed for case 2. Cases 3, 4, and 5 show very significant deviations from unity in RAIC with minimum values of $0.39,0.47$, and 0.20 respectively. Test case 6 is showing a trend with similar characteristics to case 1 , however it is more pronounced and has a minimum value of 0.66 . 


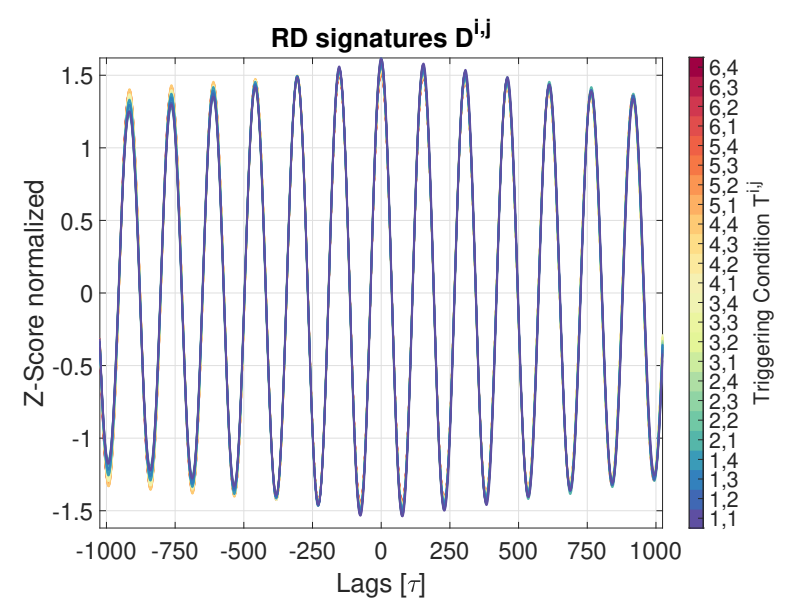

(a) Case 1, no friction force level.

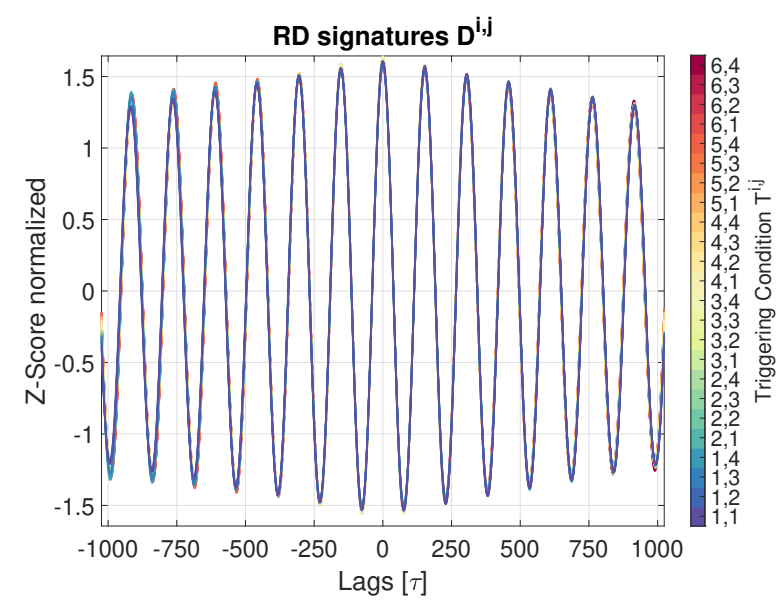

(b) Case 2, low friction force level.

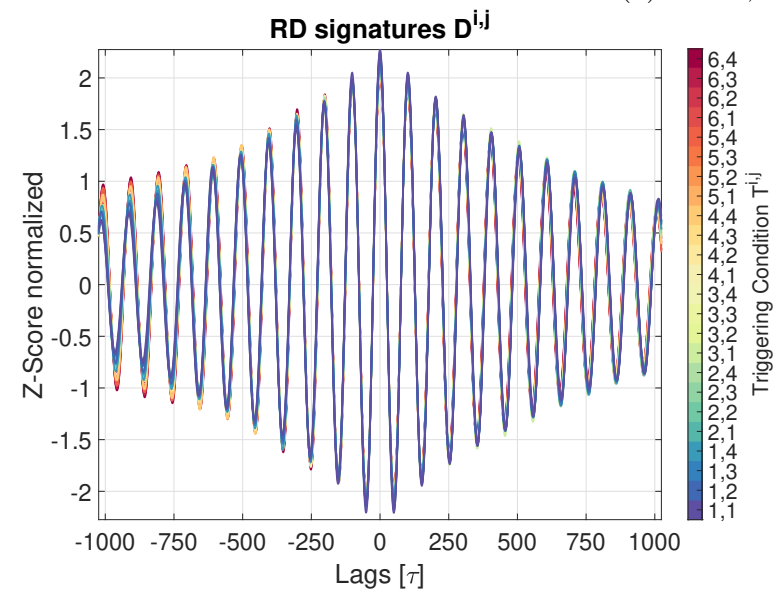

(c) Case 6, high friction force level.

Figure 9: Random decrement signatures for experimental test cases 1, 2 and 6, as described in table 4 Colorbar specify indices for the parameters in TC $T^{i, j}$ applied to calculate corresponding RD signature. If all RD signatures overlap - it is an indication of linear behavior. It not all RD signatures overlap, it is an indication of nonlinear behavior.

\subsubsection{Outcome of analysis of experimental case}

Based on the variance and the standard deviation explained by $\mathrm{PC}_{1}$ and the $\mathrm{SIC}$, case 1 could be concluded to exhibit linear behavior. However, to support this, a trend should not be present in the RAIC plot. The trend is weak, and it is therefore concluded that case 1 exhibits a slightly nonlinear behavior related to the damping. Since no friction is induced in the system, this nonlinearity must originate elsewhere. Bolted joints are known to violate the assumption of viscous damping and this could be the source of nonlinear behavior, but this has not been investigated in the present study. Another source of the nonlinear behavior could be the brush used to excite the system. Since the indication is small, we contribute it to slight imperfections in the test setup.

In case 2 , the standard deviation explained by $\mathrm{PC}_{1}$ is close to $93 \%$, and the variance explained by $\mathrm{PC}_{1}$, both just below the threshold. There is no sign of nonlinear behavior from SIC or RAIC. This case is therefore concluded to have linear behavior. This is not consistent with a low friction force level, where a slight damping nonlinearity was expected.

All 4 parameters from the analysis reveal nonlinear behavior in case 3 . The slight deviation from unity in SIC indicates that the nonlinearity is affecting the frequency of the system slightly. A significant nonlinear behavior in damping is characterized from the RAIC. 


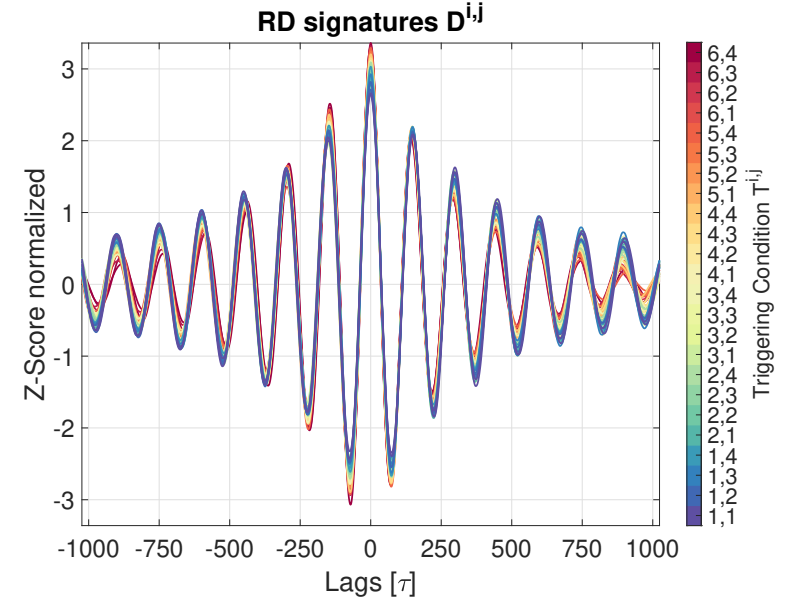

(a) Case 3, intermediate friction force level.

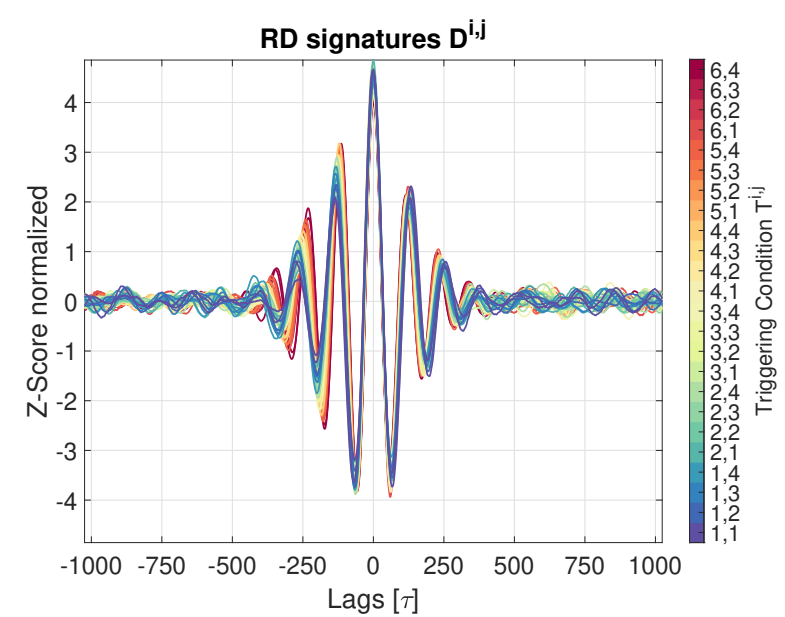

(b) Case 4, intermediate friction force level.

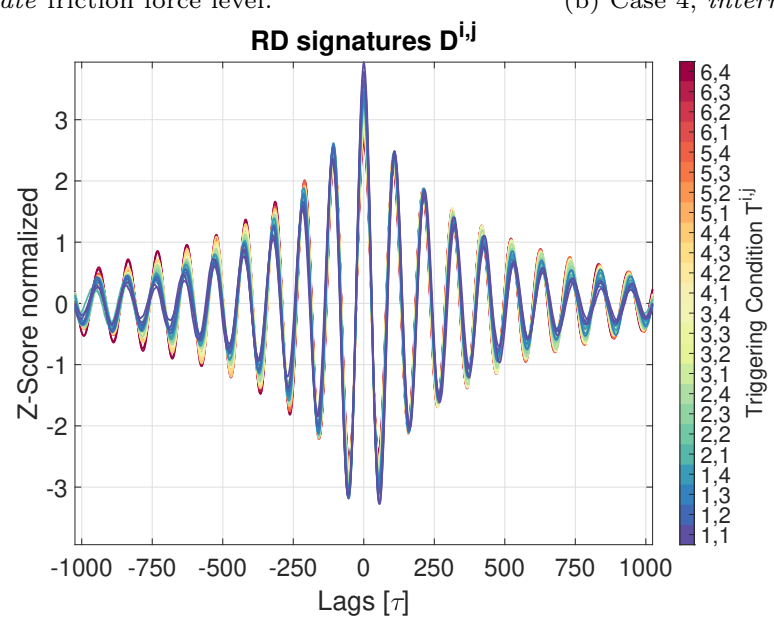

(c) Case 5, intermediate friction force level.

Figure 10: Random decrement signatures for experimental test cases 3,4 and 5, as presented in table 4 Colorbar specify indices for the parameters in $\mathrm{TC} T^{i, j}$ applied to calculate corresponding RD signature. If all RD signatures overlap - it is an indication of linear behavior. It not all RD signatures overlap, it is an indication of nonlinear behavior.
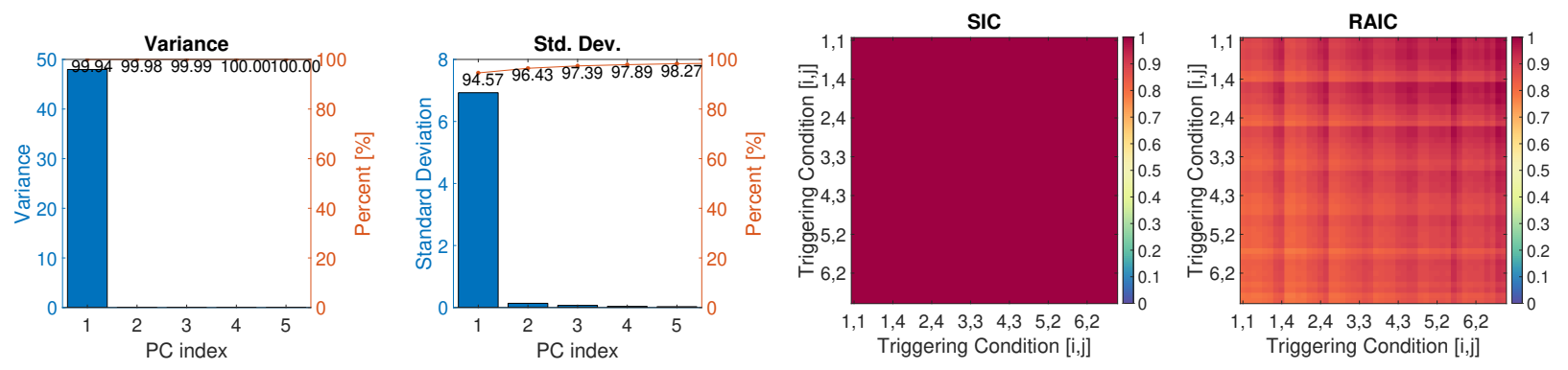

(a) Case 1, no friction force level.

Case 4 is concluded to exhibit nonlinear behavior based on all four parameters. The behavior is characterized to be related to both frequency and damping based on SIC and RAIC.

Case 5 is concluded to exhibit nonlinear behavior based on all four parameters. The nonlinear behavior is characterized to being related to both frequency and damping based on SIC and RAIC.

Case 6 is concluded to exhibit nonlinear behavior based on all parameters. The slight deviation from 

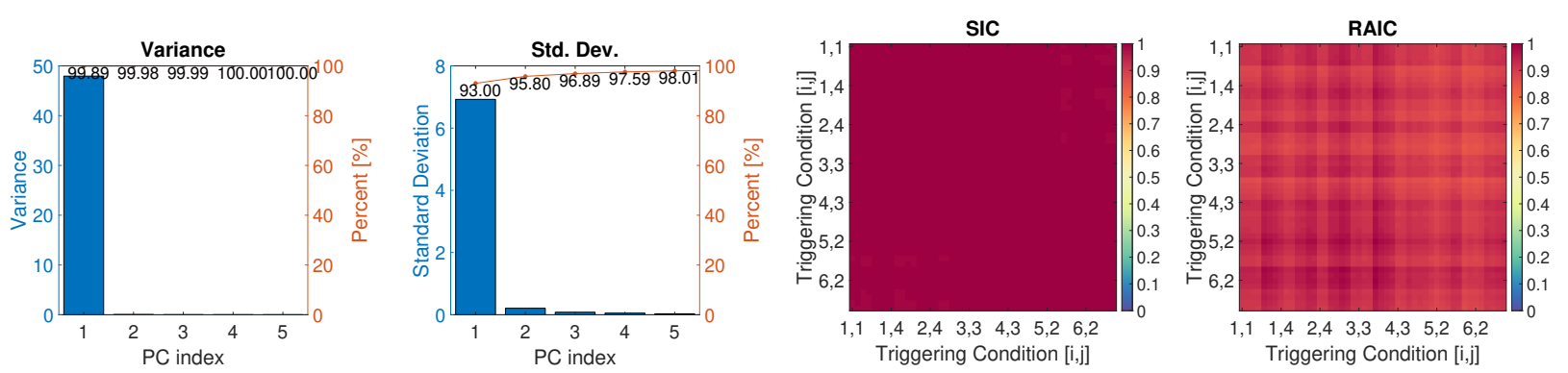

(b) Case 2, low friction force level.
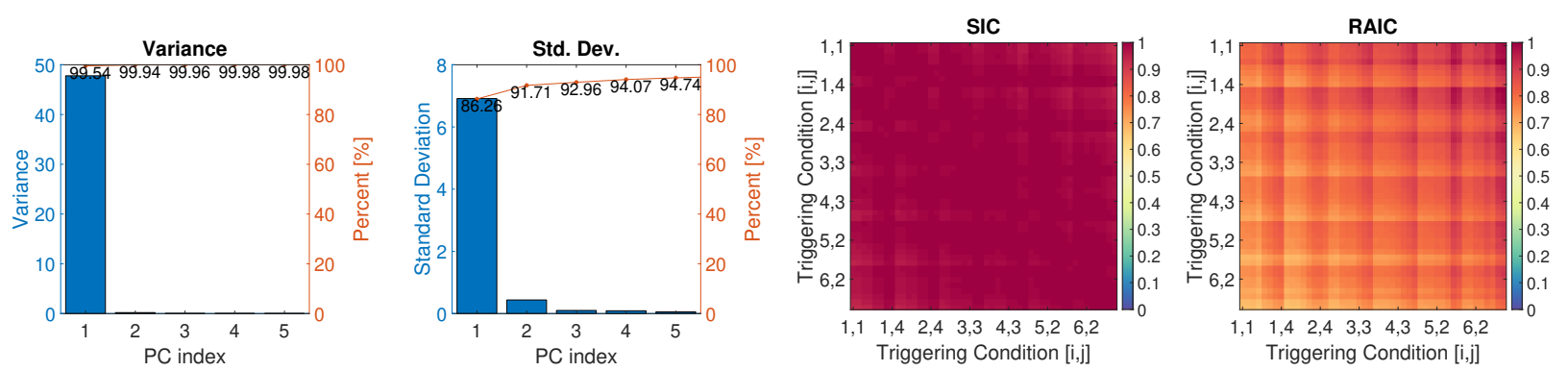

(c) Case 6, high friction force level.

Figure 11: Outcome of analysis procedure from Figure 2 applied to experimental test cases 1,2 and 6 described in Table 4 one row of plots per test case. From the left, Pareto charts for variance and standard deviation are interpreted by comparing $\mathrm{PC}_{1}$ with the thresholds defined in section 2.3 to be $\eta_{\text {thres }}=99.9 \%$ and $\gamma_{\text {thres }}=94 \%$ for the variance and standard deviation respectively. Continuing to the left SIC and RAIC summarize the parameterization of RD signatures. For both SIC and RAIC, a value less than 1 corresponds to a pair of RD signatures being dissimilar. Values in the SIC or RAIC plots that are significantly less than 1 are interpreted as nonlinear behavior related to frequency or damping respectively.

unity in SIC indicates that the nonlinearity is affecting the frequency of the system slightly. A significant nonlinear behavior in damping is characterized from the RAIC. It was expected that case 6 would exhibit linear behavior. The source of the nonlinear behavior is likely to stem from a POM-felt interaction. As the structure vibrates, the POM cylinder is pressing into the felt with a vibrating motion. This causes the felt to compress and decompress at the contact points dissipating energy. The source of the nonlinear behavior related to the frequency in this case is unknown, but could stem from the lab fixture holding the upper rod through the coupling made by the high friction force.

\section{Conclusions}

Determining if a structure is exhibiting linear or nonlinear behavior is investigated from a free decay perspective. Nonlinear behavior is recognized as instances when the free decay of a single-degree-of-freedom
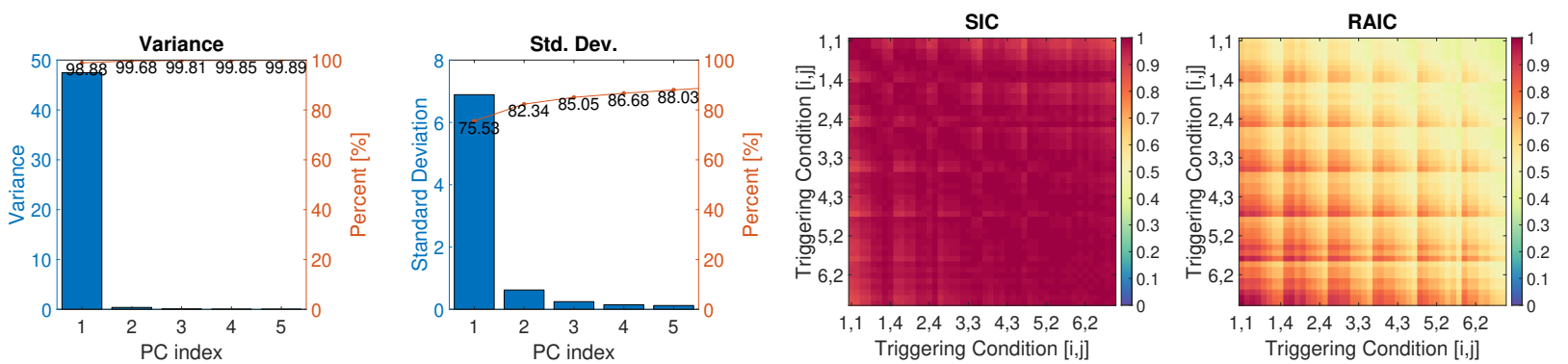

(a) Case 3, intermediate friction force level. 

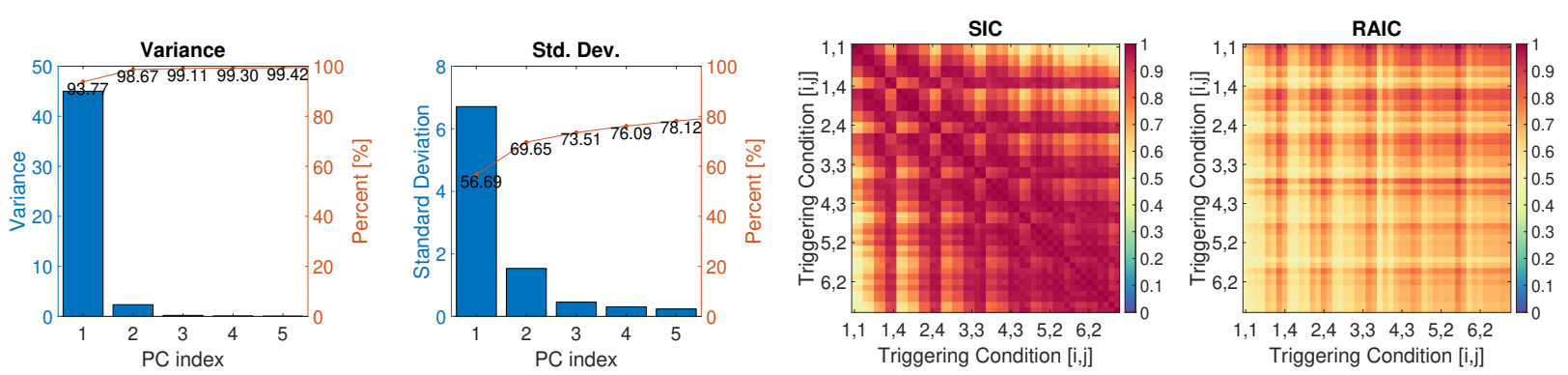

(b) Case 4, intermediate friction force level.
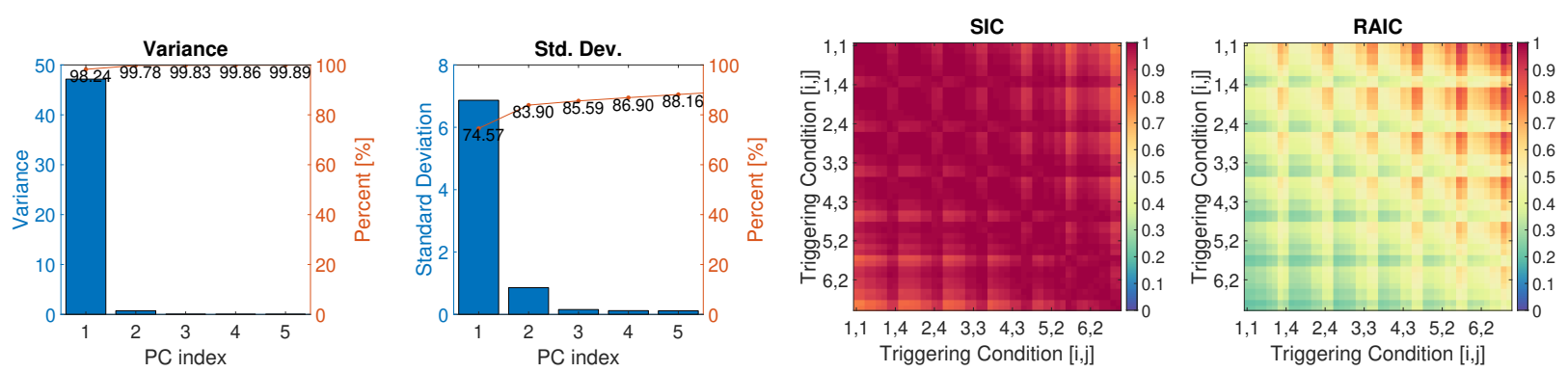

(c) Case 5, intermediate friction force level.

Figure 12: Outcome of analysis procedure from Figure 2 applied to experimental test cases 3,4 and 5 described in Table 4 one row of plots per test case. From the left, Pareto charts for variance and standard deviation are interpreted by comparing $\mathrm{PC}_{1}$ with the thresholds defined in section 2.3 to be $\eta_{\text {thres }}=99.9 \%$ and $\gamma_{\text {thres }}=94 \%$ for the variance and standard deviation respectively. Continuing to the left SIC and RAIC summarize the parameterization of RD signatures. For both SIC and RAIC, a value less than 1 corresponds to a pair of RD signatures being dissimilar. Values in the SIC or RAIC plots that are significantly less than 1 are interpreted as nonlinear behavior related to frequency or damping respectively.

system is dependent on its initial condition. An analysis method consisting of extracting and analyzing a set of random decrement $(\mathrm{RD})$ signatures with properties similar to free decays for a set of initial conditions is proposed. The detection of nonlinear behavior is realized using principal component analysis (PCA) with easily interpretable results in the form of Pareto charts for the variance and the standard deviation. Characterizing the nonlinear behavior as frequency or damping related is done using the parameters; shape invariance criterion (SIC), and relative area invariance criterion (RAIC) respectively. The procedure for detection and characterization of nonlinear behavior presented in this study can be applied to any SDOF system without any prior knowledge about the mechanical system parameters, or the input force. In future research, more sophisticated multivariate statistical methods should be applied to analyze the set of RD signatures. This could extract information about how the amplitude level and the slope of the signal at the triggering points affects the signatures, and thereby give a better characterization of the nonlinear behavior.

\section{Acknowledgements}

The authors acknowledge the funding received from Centre for Oil and Gas - DTU/Danish Hydrocarbon Research and Technology Centre (DHRTC).

\section{References}

\section{References}

[1] R. Brincker, C. Ventura, Introduction to operational modal analysis, John Wiley \& Sons, 2015.

[2] A. Brandt, Noise and vibration analysis: signal analysis and experimental procedures, John Wiley \& Sons, 2011.

[3] J. S. Bendat, A. G. Piersol, Random data: analysis and measurement procedures, Vol. 729, John Wiley \& Sons, 2011. 
[4] T. Friis, M. Tarpø, E. I. Katsanos, S. Armador, R. Brincker, Operational modal analysis based linear system identification of systems with elasto-perfectly-plastic nonlinear behavior, in: International Operational Modal Analysis Conferece (IOMAC), 2019.

[5] G. Kerschen, K. Worden, A. F. Vakakis, J.-C. Golinval, Past, present and future of nonlinear system identification in structural dynamics, Mechanical systems and signal processing 20 (3) (2006) 505-592.

[6] H. A. Cole, On-the-line analysis of random vibrations, in: AIAA/ASME 9th Structural Dynamics Materials Conference, Palm Springs, USA, 1968.

[7] H. A. Cole, Failure Detection of a Space Shuttle Wing Flutter by Random Decrement, NASA, TMX-62,041 (1971).

[8] H. A. Cole, On-line failure detection and damping measurement of space structures by random decrement signatures, NASA CR-2205 (1973).

[9] J. K. Vandiver, A. B. Dunwoody, R. B. Campbell, M. F. Cook, A mathematical basis for the random decrement vibration signature analysis technique, Journal of Mechanical Design 104 (2) (1982) 307-313.

[10] S. R. Ibrahim, Random Decrement Technique for Modal Identification of Structures, Journal of Spacecraft and Rockets 14 (11) (1977) 696-700.

[11] J. C. Asmussen, Modal analysis based on the random decrement technique: application to civil engineering structures, Ph.D. thesis, University of Aalborg (1997).

[12] A. P. Jeary, Establishing non-linear damping characteristics of structures from non-stationary response time-histories, The Structural Engineer 70 (1992) 61-6.

[13] Y. Tamura, S.-y. Suganuma, Evaluation of amplitude-dependent damping and natural frequency of buildings during strong winds, Journal of wind engineering and industrial aerodynamics 59 (2) (1996) 115-130.

[14] O. Shiryayev, J. Slater, Application of the Random Decrement Technique to a Nonlinear Dynamic System, in: 47th AIAA/ASME/ASCE/AHS/ASC Structures, Structural Dynamics, and Materials Conference, 2006 , p. 1883.

[15] Y. Tamura, Amplitude dependency of damping in buildings and critical tip drift ratio, International Journal of High-Rise Buildings 1 (1) (2012) 1-13.

[16] K. K. Vesterholm, R. Brincker, A. Brandt, Linearization of modal parameters in Duffing oscillator using the random decrement technique, in: Proceedings of the International Conference on Noise and Vibration Engineering (ISMA), 2018.

[17] K. K. Vesterholm, T. Friis, E. Katsanos, R. Brincker, A. Brandt, Output-only Estimation of Amplitude Dependent Friction-Induced Damping, in: International Modal Analysis Conference (IMAC) XXXVII, 2019.

[18] K. K. Vesterholm, T. Friis, R. Brincker, A. Brandt, Identification of stick-slip friction in single-degree of freedom system using the random decrement technique, in: Proceedings of 9th International Conference on Structural Health Monitoring of Intelligent Infrastructure, 2019.

[19] E. G. Macıas, R. Castro-Triguero, R. Gallego, J. Carretero, M. Gómez-Casero, Operational Modal Analysis and Detection of Non-linear structural behavior of bowstring Arch bridge., in: International Operational Modal Analysis Conference, 2015.

[20] K. K. Vesterholm, R. Brincker, A. Brandt, Detection of nonlinear behavior using the random decrement technique, in: International Operational Modal Analysis Conference (IOMAC), 2019.

[21] T. Friis, K. K. Vesterholm, E. I. Katsanos, A. Brandt, R. Brincker, Identification of friction-coupled offshore platforms by output-only method, in: The 29th International Ocean and Polar Engineering Conference, 2019.

[22] G. R. Tomlinson, K. Worden, Nonlinearity in structural dynamics: detection, identification and modelling, Institute of Physics Publishing, 2001.

[23] J. P. D. Hartog, Mechanical Vibrations, McGraw-Hill Book Company, Inc., New York, 1956.

[24] J. S. Bendat, Nonlinear systems techniques and applications, Wiley, 1998.

[25] R. Brincker, S. Krenk, P. H. Kirkegaard, A. Rytter, Identification of dynamical properties from correlation function estimates, Bygningsstatiske Meddelelser 63 (1) (1992) 1-38.

[26] K. Pearson, On lines and planes of closest fit to systems of points in space, The London, Edinburgh, and Dublin Philosophical Magazine and Journal of Science 2 (11) (1901) 559-572.

[27] H. Hotelling, Analysis of a complex of statistical variables into principal components., Journal of educational psychology 24 (6) (1933) 417.

[28] R. J. Allemang, D. L. Brown, A correlation coefficient for modal vector analysis, in: Proceedings of the 1st international modal analysis conference, Vol. 1, SEM Orlando, 1982, pp. 110-116.

[29] C. S. Chang, Study of dynamic characteristics of aeroelastic systems utilizing randomdec signatures, National Aeronautics and Space Administration (1975).

[30] G. M. Jenkins, Analysis of the stress-strain relationships in reactor grade graphite, British Journal of Applied Physics 13 (1) (1962) 30.

[31] K. Ahlin, M. Magnevall, A. Josefsson, Simulation of forced response in linear and nonlinear mechanical systems using digital filters, in: International Conference on Noise and Vibration Engineering (ISMA2006), K, 2006. 